\title{
Self-Organization of Inflation Volatility
}

\author{
Makoto Nirei \\ José A. Scheinkman \\ University of Tokyo \\ Columbia University
}

February 15, 2019

\begin{abstract}
We present a state-dependent pricing model that generates inflation fluctuations from idiosyncratic shocks on firms. A firm's nominal price increase lowers the other firms' relative prices, thereby inducing those firms' nominal price increases. This snow-ball effect of repricing causes the fluctuations of aggregate price without exogenous aggregate shocks. The fluctuations caused by this mechanism are more volatile when the density of firms at repricing threshold is high, and the density at the threshold is high when the trend inflation level is high. Thus, the model implies that the higher trend inflation causes the larger volatility of short-term inflation rates. Analytical and numerical analyses show that the model can account for the positive relationship between inflation level and volatility that has been observed empirically.
\end{abstract}

\section{Introduction}

Monthly aggregate prices exhibit chronic fluctuations, but the aggregate shocks that drive the aggregate prices are often elusive. Macroeconomic models treat them as 
stochastic macro-level shocks as technology shocks or monetary policy shocks. However, empirical counterparts of those shocks have been identified largely as residuals. The mechanism that generates those aggregate shocks has been of great interest in macroeconomics.

An interesting idea has been proposed that aggregate shocks can be generated from idiosyncratic shocks. Macroeconomy is replete with variety of micro-level shocks. Traditional arguments point out that the direct aggregation of the independent shocks do not add up to aggregate fluctuations due to the law-of-large-numbers effects. However, the recent literature demonstrates that the micro shocks may amount to macro shocks in an economy with highly asymmetric agents. The key is the power-law tail distributions: when the effect of idiosyncratic shocks is fat-tailed, micro shocks on a handful of extremely large agents may affect the macro variables significantly. Gabaix [18] made this point as "granular" effects by referring to a power-law distribution of firm size. Acemoglu et al. [1] used a power-law distribution of influences exercised by an industry through input-output relations.

The granular hypothesis implies that the origin of aggregate shocks should be identifiable as a set of micro shocks on large firms or key industries. However, estimates along this line seem to leave the majority of aggregate shocks still elusive. We note that the break of the law of large numbers is possible not only by the asymmetric size of micro agents but also from correlations of their actions. A familiar example is autocorrelation in time series: the law of large numbers holds for the time-average of autoregressive processes, while it may not for the processes with an autocovariance function declining as a power function.

This mechanism is introduced to macroeconomics by Bak et al. [6] and Scheinkman and Woodford [28] who consider a lattice network of supply chains. Each firm has 
inventory that acts as a buffer to sales shocks. When the inventory hits the bottom, the firm produces goods and repletes the inventory. The inventory profile converges to a distribution at which the correlation function of firms' productions with respect to the supply-chain distance declines only in power. Thus, the economy self-organizes to the inventory profile at which the aggregate fluctuations emerge endogenously. This mechanism can be extended in a general equilibrium model as shown by Nirei [25, 26]. This approach shows that, even though a macroeconomy is full of idiosyncratic shocks and micro-level interactions, it is not that any interactions are capable of generating aggregate fluctuations. Only under particular alignments, the idiosyncratic shocks and interactions lead to power laws of correlations and thus aggregate fluctuations. Therefore, this approach provides a way to identify the dimensions of macroeconomy that are potent to lead to endogenous aggregate fluctuations.

In this paper, we apply the self-organization model for inflation fluctuations that are generated endogenously from firm-level interactions. Firms set their prices depending on their competitors' pricing and incurring adjustment costs. A firm's upward repricing reduces all the competitors' relative prices, inducing them to reprice upward. Thus, firms' pricing behaviors are complementary. We show that, under the environment when a firm's adjustment induces on average one firm to adjust, the total number of adjusting firms follows a power-law distribution. Therefore, the aggregate price level exhibits significant fluctuations even if there is no aggregate shock.

This model generates an implication that a high inflation in the long run causes a high volatility in the short-run inflation. Empirical literature has generally accepted that the level and volatility of inflation rates are positively related. Since Okun [27], this relationship has been confirmed repeatedly such as by Judson and Orphanides [21] and Dincer and Eichengreen [15] who controlled for central bank independence 
and openness to international trades. In our model, the strength of the propagation effect that generates the price fluctuations depends on the average number of firms that are induced to adjust their prices when a firm adjusts. The average number of affected firms tends to one when the trend inflation rate is raised unboundedly, leading to the limiting case where the total number of adjusting firms follows a pure power law distribution. For bounded rates of inflation, the average number of affected firms is increasing in the trend inflation rate. This relation is obtained because the stationary density at the threshold is increasing in the stationary inflation rate. This implies that a higher inflation leads to a higher density at the extensive margin of price adjustments. The higher density in turn leads to the stronger propagation effect of firms' price adjustments, resulting in a higher volatility of aggregate price adjustments.

There is an extensive literature on the inflation volatility. The empirical observation on the positive association between volatility and trend inflation is an important background for central banks to set their inflation target to the low inflation range. Common wisdom is to target the annual inflation rate at about $2 \%$, and even researchers who advocate higher inflation targets do not suggest inflation rates higher than $4 \%$ (e.g., Ball [9]), because the higher inflation rate would bring about social costs. Along with redistribution effects of inflation, the greater dispersion of relative prices and the greater volatility or uncertainty of the inflation rates are commonly cited as such costs (Fischer and Modigliani [17]). The connection between the relative price dispersion and inflation is well established and its welfare implications are extensively discussed (see, e.g., Golosov and Lucas [20]; Burstein and Hellwig [10]; Nakamura and Steinsson [24]). In contrast, the positive association between inflation level and volatility has been little explored in state-dependent pricing models. Earlier work has focused on the possibility that monetary authority chooses the combination of high-level, high- 
volatility inflation (see Ball [7] and references therein). A New Keynesian explanation was provided by Ball, Mankiw and Romer [8]. In their model, a high level of inflation induces frequent repricing and more flexible aggregate prices, under which exogenous demand shocks generate large volatility of aggregate price and small volatility of output. An implication of the model is that high inflation brings about social benefits rather than costs.

The issue of trend inflation has been investigated mostly in a time-dependent pricing model subsequently, as recently surveyed by Ascari and Sbordone [5]. However, the lack of microfoundation for repricing frequency in the time-dependent models has been a serious issue in comparative statics and policy analysis. The state-dependent pricing model, which provides the microfoundation, has been beyond analytical investigations because the model loses tractability quickly.

In this context, we use a state-dependent pricing model similar to Dotsey, King and Wolman [16] and Golosov and Lucas [20]. ${ }^{1}$ We will demonstrate that the complementarity of repricing behavior between firms can lead to sizable aggregate price fluctuations arisen from idiosyncratic shocks, and furthermore, that the volatility of the aggregate price is positively associated with the long-run inflation level.

The rest of the paper is organized as follows. Section 2 builds a tractable statedependent pricing model with linear production and solves for the pricing policy and the stationary distribution of relative prices when the number of firms tends to infinity. We show the unique existence of the stationary equilibrium for each long-run inflation level

\footnotetext{
${ }^{1}$ The Golosov-Lucas model spawned empirical investigations by Midrigan [23] and Gagnon [19]. These studies pointed to an empirical pattern of threshold pricing behavior that repricing occurs when the current price deviates from the desired price. A key mechanism of this study for endogeneous price fluctuations is that a high level of inflation causes that more firms located at the lower threshold. This is supported by Gagnon [19].
} 
and conduct key comparative statics analytically. Section 3 demonstrates that, under a finite number of firms, the model generates substantial fluctuations of inflation rates around the long-run level. Key to the analysis is the degree of complementarity between firms' repricing behavior, which generates a power-law distribution of the propagation size and slows down the cancelling out effects due to the law of large numbers. Utilizing the comparative statics that a high inflation leads to a high degree of complementarity, we establish that the high inflation causes the high volatility in inflation. Section 4 calibrates the model parameters and numerically investigates the positive association between the inflation level and volatility for a wide range of inflation levels. Section 5 concludes.

\section{Model}

\subsection{Setup}

This section presents a dynamic general equilibrium model with menu costs. The

model is a continuous-time, continuous-state version of Dotsey et al. [16] with linear production. The stationary equilibrium of the model allows for comparative statics analytically under an infinite number of firms and without aggregate shocks. In particular, equilibrium real wage turns out to be decreasing in trend inflation rate, reflecting the misallocation effect of the relative price dispersion.

Consider that there are $n$ firms, each of which uses other firms' goods as inputs. Each firm $i$ supplies a differentiated intermediate good monopolistically. The intermediate $\operatorname{good} i$ is produced using labor $l_{i, t}$ linearly,

$$
y_{i, t}=l_{i, t}
$$


A competitive sector produces composite goods using the intermediate goods as

$$
Y_{t}=\left(\sum_{i=1}^{n} y_{i, t}^{(\eta-1) / \eta} n^{-1 / \eta}\right)^{\eta /(\eta-1)}
$$

where $\eta>1$ is the elasticity of substitution. Let $P_{t}$ denote the price of the final goods. Then, the optimization condition for the production of final goods leads to a demand function for intermediate goods as $y_{i, t}=p_{i, t}^{-\eta} Y_{t} / n$, where $p_{i, t} \equiv P_{i, t} / P_{t}$ denotes the relative price of good $i$. The price of the final goods is $P_{t}=\left(\sum_{i=1}^{n} P_{i, t}^{1-\eta} / n\right)^{1 /(1-\eta)}$. Thus, the inflation rate of consumer price index in this model is $\pi_{t}=\left(d P_{t} / d t\right) / P_{t}$.

Let $W_{t}$ denote the nominal wage rate and $w_{t} \equiv W_{t} / P_{t}$ the real wage that firm $i$ faces. The firm's real profit function is

$$
Z\left(p_{i, t}, w_{t}, Y_{t}\right)=\left(p_{i, t}^{1-\eta}-w_{t} p_{i, t}^{-\eta}\right) Y_{t} / n
$$

Firm $i$ chooses its price $p_{i, t}$ to maximize the discounted value of the firm's future profit stream. There is no capital in this economy. The prices of intermediate goods are sticky. We assume that the firm can adjust the price of its good by bearing adjustment $\operatorname{costs} \delta Y_{t} / n$. We choose this specification of the adjustment cost so that the firms' pricing solution is explicitly derived. Let $\lambda_{t, t+d t}$ denote a fraction of firms that incur repricing costs in time interval $[t, t+d t)$. Also, $\lambda$ denotes the stationary rate at which a firm incurs repricing costs. Thus, $\lambda_{t, t+d t}=\lambda d t$ for any $t$ at a stationary equilibrium.

In addition, we assume that the firms draw a chance with Poisson rate $\mu$ at which they can adjust prices without paying adjustment costs. Thus, this model includes both elements of the state-dependent pricing and the Calvo pricing models, as in Stokey [30]. The Calvo shock is the only exogenous shock in this model. Thus, the Calvo shock represents all idiosyncratic disturbances on pricing conditions such as adjustment costs, marginal costs and productivity. By this simplification, our model gains tractability needed to characterize aggregate price fluctuations. 
Households play a minor role in this model. Composite good $C_{t}$ is consumed by representative households whose preference is given by $\int_{0}^{\infty} e^{-\rho t} U\left(C_{t}, N_{t}\right) d t$, where $\rho$ denotes the time discount rate and $N_{t}$ labor supply. The labor market is competitive. Combining the goods demand function and the labor demand function yields $p_{i, t}^{-\eta} / n=$ $y_{i, t} / Y=l_{i, t} / Y$, leading to

$$
Y_{t}=N_{t} /\left(\sum_{i=1}^{n} p_{i, t}^{-\eta} / n\right)
$$

at the labor market equilibrium $\sum_{i=1}^{n} l_{i, t}=N_{t}$.

We consider the stationary equilibrium where the aggregate price $P_{t}$ grows at a stationary inflation rate $\pi$ for any $t$. Representative households request firms to maximize their sum of future profits discounted at rate $\rho$. The intra-temporal optimality condition for households is $U_{N}=-U_{C} w$. The market clearing condition for final goods is

$$
Y=C+\lambda \delta Y .
$$

Given $w$ and the stationary distribution of $p_{i, t}$, the steady-state output $Y$ is determined by these conditions.

Firms' repricing plan is characterized by a threshold rule, because they incur fixed adjustment costs. We focus on the case of positive inflation rate $\pi>0$. The deflationary case $\pi<0$ can be symmetrically analyzed in our framework. Under $\pi>0$, the optimal pricing policy takes the form of a one-sided regulator, in which a firm reprices upward when it reaches a lower threshold, denoted by $\underline{p}$. An interval $\left(\underline{p}, p^{*}\right]$ forms a firm's inaction region where it is optimal for the firm not to adjust the price unless the firm draws a Calvo shock.

Consider a firm at $t=0$, and let $T$ denote the time when the firm reprices by incurring adjustment $\operatorname{cost} \delta$ and $\tau$ the time when the firm draws a Calvo shock. Let $p^{*}$ denote the relative price which the firm reverts to when it adjusts the price. The 
firm's value function is formulated as

$$
\begin{aligned}
V\left(p_{0}, w_{0}, Y_{0}\right)= & \sup _{\left\{p^{*}, T\right\}}\left\{\operatorname{Pr}(\tau>T)\left(\int_{0}^{T} e^{-\rho t} Z\left(p_{t}, w_{t}, Y_{t}\right) d t+e^{-\rho T}\left(V\left(p^{*}, w_{T}, Y_{T}\right)-\delta Y_{T} / n\right)\right)\right. \\
& \left.+\int_{0}^{T} \operatorname{Pr}(\tau=T)\left(\int_{0}^{\tau} e^{-\rho t} Z\left(p_{t}, w_{t}, Y_{t}\right) d t+e^{-\rho \tau} V\left(p^{*}, w_{\tau}, Y_{\tau}\right)\right) d \tau\right\} .
\end{aligned}
$$

Within the inaction band, relative price $p_{t}$ declines exogenously by inflation. At the steady state, $w_{t}$ and $Y_{t}$ are constant, and the threshold rule depends only on $p_{t}$. Note that the value function $V$ is linear in $Y$ at the steady state, since $Y$ linearly affects the adjustment cost and profits (2). Thus, the optimal pricing policy $\left(p^{*}, T\right)$ is not affected by $Y$. The profit function (2) can be normalized by the production level at the steady state as

$$
z \equiv \frac{Z}{Y / n}=p^{1-\eta}-w p^{-\eta}
$$

and the normalized value function $v \equiv V /(Y / n)$ is written as

$$
\begin{aligned}
v\left(p_{0}\right)= & \sup _{\left\{p^{*}, T\right\}}\left\{e^{-\mu T}\left(\int_{0}^{T} e^{-\rho t} z\left(p_{t}\right) d t+e^{-\rho T}\left(v\left(p^{*}\right)-\delta\right)\right)\right. \\
& \left.+\int_{0}^{T} \mu e^{-\mu \tau}\left(\int_{0}^{\tau} e^{-\rho t} z\left(p_{t}\right) d t+e^{-\rho \tau} v\left(p^{*}\right)\right) d \tau\right\} .
\end{aligned}
$$

using that $\tau$ follows an exponential distribution with mean $1 / \mu$. Note that choosing $T$ is equivalent to choosing $\underline{p}$.

A stationary equilibrium when $n$ tends to infinity is defined as aggregate variables $(\pi, w, Y, C, N, \lambda)$, the distributions of $\left(p_{i, t}, y_{i, t}, l_{i, t}\right)_{i, t}$, the value function $v$ and pricing policy $\left\{p^{*}, \underline{p}\right\}$, and the path of aggregate price $P_{t}$ that satisfy the following conditions: (i) the allocation maximizes the household's utility given prices, (ii) the value and policy functions, allocation and goods prices solve the firms' dynamic optimization problem given prices, (iii) markets clear for the final good, intermediate goods and labor, (iv) the distribution of relative prices $p_{i, t}$ is stationary, and (v) $P_{t}$ grows at a constant rate $\pi$. 


\subsection{Comparative statics of stationary equilibrium}

First, we derive firms' optimality conditions at the stationary equilibrium. For $p_{0}$ outside of inaction region $\left(\underline{p}, p^{*}\right), T=0$ holds, and thus $v\left(p_{0}\right)=v\left(p^{*}\right)-\delta$. Inside inaction region $p_{0} \in\left(\underline{p}, p^{*}\right)$, the relative price evolves as $d p_{t} / d t=-\pi p_{t}$, and the Hamilton-Jacobi-Bellman equation is obtained as

$$
(\rho+\mu) v(p)=\sup _{p^{*}}\left[z(p)+\mu v\left(p^{*}\right)-\pi p v^{\prime}(p)\right] .
$$

Note that the repricing point $p^{*}$ does not depend on the current relative price $p$ in the right-hand side. Thus, we can differentiate the both sides of (6) with respect to $p$ with taking $\sup _{p^{*}} \mu v\left(p^{*}\right)$ as an unknown constant, resulting in $(\rho+\mu) v^{\prime}(p)=z^{\prime}(p)-\pi v^{\prime}(p)-$ $\pi p v^{\prime \prime}(p)$. Evaluating this at $p^{*}$ such that $v^{\prime}\left(p^{*}\right)=0$, we obtain $z^{\prime}\left(p^{*}\right)=\pi p^{*} v^{\prime \prime}\left(p^{*}\right)$. Now let $\hat{p} \equiv(\eta /(\eta-1)) w$ denote the price which maximizes a static profit: $z^{\prime}(\hat{p})=0$. Then, $z^{\prime}(p)=-\eta p^{-\eta-1}(((\eta-1) / \eta) p-w)$ is strictly negative for $p>\hat{p}$. Therefore, $v^{\prime \prime}\left(p^{*}\right)<0$ if $p^{*}>\hat{p}$, where the inequality $p^{*}>\hat{p}$ holds true as shown shortly. Thus, the value

function is locally strictly concave at $p^{*}$ such that $v^{\prime}\left(p^{*}\right)=0$, implying that such $p^{*}$ achieves the supremum of $\mu v\left(p^{*}\right)$. Evaluating (6) at $p^{*}$, we obtain $\rho v\left(p^{*}\right)=z\left(p^{*}\right)$.

Given $p^{*}$ Equation (6) is a first-order linear ordinary differential equation up to an unknown constant $\mu v\left(p^{*}\right)=z\left(p^{*}\right) \mu / \rho$, which has a class of solution

$$
v(p)=c_{0} p^{-(\rho+\mu) / \pi}+\frac{p^{1-\eta}}{\rho+\mu-\pi(\eta-1)}-\frac{w p^{-\eta}}{\rho+\mu-\pi \eta}+\frac{z\left(p^{*}\right) \mu / \rho}{\rho+\mu} .
$$

with unknown coefficient $c_{0}$.

The firm's optimal choice for $p^{*}$ and $\underline{p}$ leads to the value matching condition

$$
v\left(p^{*}\right)-\delta=v(\underline{p})
$$


and the first order conditions for $p^{*}$ and $\underline{p}$

$$
\begin{aligned}
v^{\prime}\left(p^{*}\right) & =0, \\
z(\underline{p}) & =\rho v\left(p^{*}\right)-(\rho+\mu) \delta .
\end{aligned}
$$

The condition (10) is derived by the first order condition for the maximization (5) with respect to $T$. By adjusting the price at a marginally earlier timing by $d t$ at state $\underline{p}$, or equivalently, by marginally increasing $\underline{p}$, a firm loses an instantaneous profit $z(\underline{p}) d t$ and a chance of not paying adjustment costs $\delta \mu d t$, on the one hand. On the other hand, the firm gains the discounted value of adjusting, $\left(v\left(p^{*}\right)-\delta\right) \rho d t$. Thus, the condition (10) assures that a marginal change in $\underline{p}$ does not increase the value of firm.

Conditions $(7,8,9,10)$ determine $\left\{p^{*}, \underline{p}, c_{0}, v(p)\right\}$, which solves the firms' maximization problem given $w$. Let $q \equiv p^{*} / \underline{p}>1$ denote the size of price adjustment for the firm hitting $\underline{p}$. Combining $(9,10)$ and $\rho v\left(p^{*}\right)=z\left(p^{*}\right)$, we obtain $z\left(p^{*}\right)-z(\underline{p})=(\rho+\mu) \delta$, which is rewritten as

$$
(\rho+\mu) \delta=\left(q^{1-\eta}-1\right) \underline{p}^{1-\eta}-w\left(q^{-\eta}-1\right) \underline{p}^{-\eta} \quad \text { [Value Maching]. }
$$

Moreover, the optimal markup over the marginal labor cost can be solved as follows (see Appendix A for the derivation):

$$
p^{*}=q \underline{p}=\frac{\varphi\left(q, \eta-\frac{\rho+\mu}{\pi}\right)}{\varphi\left(q, \eta-1-\frac{\rho+\mu}{\pi}\right)} \frac{\eta}{\eta-1} w \quad \text { [Optimal Markup] }
$$

where $\varphi(q, x) \equiv\left(q^{x}-1\right) / x$ is an integral of $q^{x-1}$ with respect to $q$. From this equation, it is verified that $p^{*}>\hat{p}=(\eta /(\eta-1)) w$, using that $\varphi(\cdot, \cdot)$ is strictly increasing in the second argument as shown in Appendix A. Function $\varphi$ will turn out to be useful for characterizing both the stationary distribution and the optimal pricing rule.

Next, we derive the stationary distribution of relative prices. Since $P_{t}$ grows at $\pi>0$, the firm's relative price $p_{i, t}$ deterministically declines at rate $\pi$ unless the firm 
draws a Calvo shock, while it is adjusted to $p^{*}$ when $p_{i, t}$ reaches $\underline{p}$ or a Calvo shock arrives at rate $\mu$. We define a relevant state variable $s_{i, t} \equiv\left(\log p_{i, t}-\log \underline{p}\right) / \log q$, which denotes a firm's distance from the threshold relative price, normalized by the repricing size $\log q$. Let $f(s)$ denote the distribution of $s$ over its support $(0,1]$. The state $s$ declines by $\pi / \log q$ due to inflation, while it is reset to 1 when it reaches 0 or randomly at rate $\mu$. Thus, $f(s)$ evolves according to Kolmogorov forward equation $d f(s)=f(s+(\pi / \log q) d t)-f(s)-f(s) \mu d t$. We find the stationary distribution $f(s)$ by solving $d f(s)=0$. Dividing both sides of the equation by $d t$ and taking $d t \rightarrow 0$, we obtain an ordinary differential equation $0=f^{\prime}(s) \pi / \log q-f(s) \mu$, whose solution has an exponential form. Since $f(s)$ must integrate to 1 , we obtain the solution as

$$
f(s)=f_{o} q^{s \mu / \pi}
$$

where $f_{o} \equiv \log q / \varphi(q, \mu / \pi)$ denotes the stationary density of firms at the repricing threshold $s=0$.

From $p_{i, t}=P_{i, t} / P_{t}$ and demand functions, an aggregation condition must hold:

$$
\sum_{i=1}^{n} p_{i, t}^{1-\eta} / n=1
$$

As $n \rightarrow \infty$, the left-hand side tends to $E\left[p_{i, t}^{1-\eta}\right]$ where the expectation is evaluated using the stationary distribution $f(s)$ and $p=\underline{p}\left(p^{*} / \underline{p}\right)^{s}$. Applying the stationary distribution, the above equation leads to

$$
\underline{p}^{\eta-1}=\frac{\varphi\left(q, 1-\eta+\frac{\mu}{\pi}\right)}{\varphi\left(q, \frac{\mu}{\pi}\right)} \quad \text { [Stationary Distribution]. }
$$

Hence, the pricing policy $(q, \underline{p})$ is determined given $w$ by $(11,12)$, and the stationarity condition (14) puts a restriction on the policy $(q, \underline{p})$, whereby determining $q$, $\underline{p}$, and $w$. Note that $\underline{p}$ is linear in $w$ in (12). Substituting $w$ out, and expressing 
$\underline{p}^{\eta-1} / \varphi(q, 1-\eta)$ by the optimal pricing $(11,12)$ and by stationary distribution $(14)$, we obtain the equation that determines $q$ :

$$
\frac{\eta-1}{(\rho+\mu) \delta}\left[\frac{\varphi(q,-\eta)}{\varphi(q, 1-\eta)} \frac{\varphi\left(q, 1-\eta+\frac{\rho+\mu}{\pi}\right)}{\varphi\left(q,-\eta+\frac{\rho+\mu}{\pi}\right)}-1\right]=\frac{\varphi\left(q, 1-\eta+\frac{\mu}{\pi}\right)}{\varphi\left(q, \frac{\mu}{\pi}\right) \varphi(q, 1-\eta)}
$$

The left-hand side is equal to $\underline{p}^{\eta-1} / \varphi(q, 1-\eta)$, indicating that the difference in profits between $p^{*}$ and $\underline{p}$ is determined by the repricing cost $\delta$. Thus, an increase in $q$ raises $\underline{p}$. The right-hand side expresses that the relative prices must aggregate to 1 . Thus, $q$ decreases $\underline{p}$. Using this, we establish that the solution exists uniquely.

Proposition 1. There exists a unique stationary equilibrium for each $\pi>0$.

All proofs are deferred to Appendix A unless stated otherwise. This proposition establishes the unique existence of stationary equilibrium for a state-dependent pricing model with endogenous real wage and without quadratic approximation. ${ }^{2}$ In this model, the real wage is affected by the inflation rate through optimal markup behavior, even though the marginal product of labor is constant due to the linear production technology. For the pricing behavior and real wage, we obtain the following comparative statics of the stationary equilibrium.

Proposition 2. 1. Repricing size $\log q$ increases unboundedly as $\pi$ increases.

\section{Maximum markup $p^{*} / w$ increases as $\pi$ increases.}

\footnotetext{
${ }^{2}$ In the tradition of state-dependent pricing models, analytical results are obtained by taking real wage or real cost of production exogenous (Sheshinski and Weiss [29]; Caplin and Spulber [13]; Caballero and Engel [11]; Caplin and Leahy [12]; Ahlin and Shintani [2]; Stokey [30]; Alvarez and Lippi [4]) and the general equilibrium results are obtained numerically (Dotsey et al. [16]; Golosov and Lucas [20]). An exception is Danziger [14] which analytically establishes the existence of Markov perfect equilibrium under $\eta=2$.
} 


\section{Real wage $w$ decreases as $\pi$ increases for sufficiently large $\pi$.}

The first item is well established since Sheshinski and Weiss [29], and its intuition is clear: a higher inflation causes more frequent repricing, inducing a firm to adjust its price by a higher margin in order to gain more time till the next repricing and to minimize repricing costs. The second result follows naturally in this model: a larger repricing size implies a higher upper bound of inaction region, which coincides with the reverting price when there is no idiosyncratic shock.

The third item claims that the real wage decreases as inflation increases. When the inflation is high, the relative price dispersion and the resulting inefficiency loss in the production sector are large. Thus, in a general equilibrium of good and labor markets, the real wage is decreased as a result of low efficiency in aggregate production. The result seems new in the literature in which most analytically results are obtained with

exogenous real wage. An exception is Danziger [14], in which a high inflation leads to a low markup and a high real wage. The signs are opposite to the results here, because the optimal pricing in Danziger's model is determined by option values of inaction under the presence of future idiosyncratic productivity shock, which is abstracted in this model.

The positive relation between $\pi$ and $q$ implies that a high inflation increases the intensive margin of aggregate price adjustments, which will be a factor for the effect of $\pi$ on inflation volatility. However, the main source of inflation volatility - in a causal sense as well as in terms of quantitative significance - in this model is an extensive margin, that is, the number of firms that reprice simultaneously, as we show in Section 3 . 


\subsection{Complementarity of repricing at extensive margin}

The extensive margin of aggregate price adjustments is $\lambda$, the rate of firms that reprice at the lower threshold of the inaction band. The extent of $\lambda$ is determined by the complementarity between repricing behaviors. We define $\theta$ as the mean number of firms that are induced to reprice as a result of a firm's repricing at the threshold, and call it the degree of complementarity. The degree of complementarity $\theta$ is derived as follows at the stationary equilibrium.

Suppose that firm $i$ hits the threshold $\underline{p}$ and reprices its price $\log P_{i, t}$ by $\log q$. The price change by firm $i$ increases the aggregate good price $P_{t}$, which in turn decreases the relative price of other firms $p_{j, t}, j \neq i$. The impact of $P_{i, t}$ on $P_{t}$ is computed as follows. Note that $P_{t}=\left(\sum_{i=1}^{n} e^{(1-\eta) \log P_{i, t} / n}\right)^{1 /(1-\eta)}$. Now $\log P_{i, t}$ is increased by $\Delta \log P_{i, t}$. By Maclaurin series expansion around the given initial price $\log P_{i, t}$, we obtain

$$
\begin{aligned}
\Delta \log P_{t} & =\frac{1}{n} \frac{p_{i, t}^{1-\eta}}{1-\eta} \sum_{k=1}^{\infty} \frac{\left((1-\eta) \Delta \log P_{i, t}\right)^{k}}{k !}+O\left(n^{-2}\right) \\
& =\frac{p_{i, t}^{1-\eta}}{n} \frac{e^{(1-\eta) \Delta \log P_{i, t}-1}}{1-\eta}+O\left(n^{-2}\right) \\
& =\frac{p_{i, t}^{1-\eta}}{n} \varphi(q, 1-\eta)+O\left(n^{-2}\right)
\end{aligned}
$$

Now, the increase in aggregate price reduces $s_{j, t}$ by $\Delta \log P_{t} / \log q$. Firms with $s_{j, t}$ in the small interval $\left(0, \Delta \log P_{t} / \log q\right]$ are induced to reprice by firm $i$ 's repricing. Since the stationary density at the threshold is $f_{o}$, the mean number of firms that are induced to reprice by firm $i$ is $\theta \equiv f_{o} \varphi(q, 1-\eta) \underline{p}^{1-\eta} / \log q$. Using (13) and (14), we obtain

$$
\theta=\frac{\varphi(q, 1-\eta)}{\varphi(q, 1-\eta+\mu / \pi)}
$$


The firm $j$ 's repricing further increases the aggregate price by $\Delta \log P_{t}$, decreases other firms' states by $\Delta \log P_{t} / \log q$, and induces some of them to reprice. Noting that $\theta<1$ holds for any $\pi$ by (17), the mean total number of repricing firm is determined as $1 /(1-\theta)$. Since $\mu d t$ fraction of firms incur Calvo shocks in a small time interval $d t$, the extensive margin $\lambda d t$ is determined as $\mu d t \theta /(1-\theta)$. Hence, the complementarity $\theta$ determines the extent of multiplier effects on the extensive margin $\lambda$.

This degree of complementarity $\theta$ turns out to increase as the inflation rate rises.

Proposition 3. Degree of complementarity $\theta$ is increasing in $\pi$ for sufficiently large $\pi$.

This proposition shows that the higher inflation results in the greater multiplier effects in pricing behavior. As the trend inflation rate $\pi$ increases, the stationary distribution of relative prices skews to the left. This leads to an increase in the density of firms near the repricing threshold. In this situation, a repricing action by a triggering firm causes a larger size of avalanche of repricing by other firms. Hence, a higher inflation rate generates a larger multiplier effect and results in a larger fraction of repricing firms $\lambda$. Thus we obtain the following.

Corollary 1. The stationary repricing rate $\mu+\lambda=\mu /(1-\theta)$ is increasing in $\pi$ for sufficiently large $\pi$.

This result, along with the comparative statics of $q$, is reminiscent of Sheshinski and Weiss [29]. These comparative statics between the repricing frequency $(\mu+\lambda)$ or the repricing size $(\log q)$ and the inflation rate $(\pi)$ are shown to be consistent with the empirical evidence from high-inflation episodes by Gagnon [19] and Alvarez et al. [3].

In this section, we showed that the complementarity $\theta$ leads to a multiplier effect on the mean repricing behavior. In the next section, we argue that the complementarity 
generates not only the mean multiplier effect but also volatility in aggregate price when $n$ is large but finite.

\section{Fluctuations in a finite economy}

In this section, we demonstrate that the model economy exhibits quantitatively significant fluctuations in inflation rates around the long-run level $\pi$ when the number of firms $n$ is large but finite. When $n$ is finite, idiosyncratic shocks (Calvo events) generate some aggregate fluctuations in principle, but the variance of the aggregate fluctuation vanishes quickly as $n$ tends to infinity according to the law of large numbers if the repricing behavior is independent. Whether the sum of idiosyncratic shocks matters for the aggregates depends on the degree of complementarity $\theta$ of repricing behavior. This section shows that the complementarity generates a fat-tailed distribution of the number of firms that reprice simultaneously, leading to that (i) short-term inflation rates exhibit non-negligible fluctuations and (ii) a high level of long-term inflation causes high volatility of short-term inflation rates.

\subsection{Stochastic number of firms that reprice simultaneously}

In an economy with finite $n$, any realized moment of the cross-section distribution of $s_{i, t}$ is slightly disturbed from the population moment derived from the stationary distribution $f(s)$. The aggregate price in the finite model can deviate from its stationary counterpart in the model with infinitely many firms. Because firms' pricing decisions are positively correlated, a firm's discrete adjustment in price may have substantial avalanche effects on adjustments by other firms. In this section we characterize the asymptotic property of this avalanche effect formally. 
To keep the analysis simple with the finite number of firms and fluctuating aggregate prices, we consider that the monetary authority is capable of implementing the average inflation rate at its target $\pi$ and also accommodating any shocks to realize the constant real interest rate at the household's time-discount rate $r_{t}=\rho$. We consider that households hold monetary base $M_{t}$ which yields nominal interest rate $i_{t}$. Households obey a cash-in-advance constraint $M_{t}=P_{t} C_{t}$. Households also own the firms in this economy, and obtain total nominal profits as dividend $D_{t}$. The household's flow budget constraint is written as $P_{t} C_{t}+\dot{M}_{t}=W_{t} N_{t}+D_{t}+i_{t} M_{t}$. The monetary authority increases $M_{t}$ at rate $\pi$ so that the average inflation rate meets its target $\pi$. The real interest rate is denoted by $r_{t} \equiv i_{t}-\pi_{t}$. The monetary authority absorbs any shock on $\pi_{t}$ by nominal interest rate $i_{t}$ so that the real interest rate is constant at $r_{t}=\rho$ for any $t$.

When a firm reprices due to a Calvo shock, it reduces the relative prices of all other firms, which may induce some firms near the threshold to reprice. The number of firms that reprice after the initial firm depends on the distribution of $s_{j, t}$. Let $L_{t}$ denote the total number of firms that reprice after a firm adjusts due to a Calvo shock within an instance $t$. The ratio of those firms to all firms, $L_{t} / n$, forms the stochastic extensive margin of aggregate price adjustments in a finite economy. Then, aggregate price level $\log P_{t}$ is represented as a compound Poisson process with arrival rate $\mu$ : the aggregate price level does not move when no firms draw Calvo shocks, whereas it jumps if a Calvo shock arrives at a firm, and the jump size of $\log P_{t}$ is determined by the extensive margin $L_{t} / n$ and the intensive margin $\log q$.

We make an approximation that, in the finite economy, the real macroeconomic variables $r, w$, and $Y$ stay at the stationary level and firms follow the stationary policy rule $\left(p^{*}, \underline{p}\right)$. This environment holds asymptotically as $n$ tends to infinity, because the real aggregate variables are dependent only on relative prices, and the moments 
of relative prices $\left(p_{i, t}\right)$ obey the law of large numbers even though the aggregate price inflation rates fluctuate. Thus, the deviation of relative prices from the stationary distribution has only vanishingly small impacts on real aggregate variables as $n$ diverges. The real wage rate asymptotically stays at the stationary level, since the nominal wage rate adjusts flexibly in the model. The monetary authority is able to accommodate to a jump in $P_{t}$ by adjusting monetary base $M_{t}$ instantly.

Suppose that a state profile $\left(s_{i, t}\right)_{i=1}^{n}$ is randomly drawn from the joint stationary density function $f^{n}(s)$. Suppose that firm $i$ draws a Calvo shock in $t$. The repricing of $i$ reduces $\left(s_{j, t}\right)_{j \neq i}$ and induces some firms near the threshold to reprice. Let $m_{0}$ denote the number of those repricing firms. The repricings of those $m_{0}$ firms further reduce the relative prices of other firms, inducing some firms to reprice. Let $m_{1}$ denote the number of those repricing firms. This process continues until there is no firm induced to reprice. Thus, the total number of adjusting firms within $t$ (other than firm $i$ ) is denoted by the total sum during the repricing process: $L=\sum_{u=0}^{U} m_{u}$, where $U$ is the smallest integer satisfying $m_{U}=0$. During this process, all the firms reprice at the extensive margin, so their repricing size is always $\log q$. The mean number of firms that are induced to reprice by a firm repriced by $\log q$ has been derived as $\theta$ in (17). Hence, in this adjustment process, each repricing firm gives rise to a random number of repricing firms, which follows a Poisson distribution with mean $\theta$ asymptotically as $n \rightarrow \infty$. Thus, $m_{u}$ for $u=0,1, \ldots, U$ is embedded in a so-called Poisson branching process with mean $\theta$. This allows for the following characterization of the fluctuation of the sum $L$.

Proposition 4. The asymptotic variance of $L$ as $n \rightarrow \infty$ conditional on $m_{0}=1$ is $\theta /(1-\theta)^{3}$. Furthermore, as $n \rightarrow \infty$, the distribution of $L$ conditional on $m_{0}$ asymp- 
totically follows:

$$
\begin{aligned}
\operatorname{Pr}\left(L=\ell \mid m_{0}\right) & =\frac{m_{0}}{\ell} \frac{e^{-\theta \ell}(\theta \ell)^{\ell-m_{0}}}{\left(\ell-m_{0}\right) !} \\
& \propto e^{-(\theta-1-\log \theta) \ell} \ell^{-1.5} \quad \text { as } \ell \rightarrow \infty .
\end{aligned}
$$

This proposition shows that the variance of $L$ is increasing in the degree of complementarity $\theta$. The increase is non-linear and rather rapid when $\theta$ is close to 1 . Moreover, $\theta$ is increasing in $\pi$ by Proposition 3. Hence, we obtain that the variance of the extensive margin of aggregate price adjustments increases as the long-run inflation level $\pi$ increases.

In the previous section, we showed that the degree of complementarity $\theta$ determines a multiplier effect of repricing. A firm's reprice induces $\theta$ firms to reprice on average, leading to the mean number of subsequently repricing firms to be $1 /(1-\theta)$. Proposition 4 now shows that the multiplier effect is stochastic under finite $n$, and the variance of the multiplier effect is increasing in $\theta$ as $\theta /(1-\theta)^{3}$. When $\theta$ approaches 1 as $\pi$ increases unboundedly, the variance of $L$ diverges. Therefore, Proposition 4 points to the possibility that the fluctuations of aggregate prices are obtained for any large number of firms $n$ when $\pi$ is sufficiently high.

Proposition 4 also indicates that the number of firms that reprice simultaneously has a power-law distribution with exponential truncation as shown in (19). Therefore, the multiplier effect caused by the complementarity of repricing is not only stochastic under finite $n$, but also exhibits a fat right tail, signified by the power-law distribution, up to the exponential truncation point determined by $\theta$. When $\theta$ reaches 1 , the truncation point diverges, implying that the entire tail is characterized by a power-law distribution.

The power law of the multiplier effect $L$ is the key to generate inflation fluctuations. The power-law tailed distribution for the avalanche of simultaneously repricing firms 
is reminiscent of the self-organized criticality model of inventories proposed by Bak et al. [6] and Scheinkman and Woodford [28]. In their model, the configuration of agents' states (inventory profile) globally converges to the criticality point of pairwise correlation of actions, at which the power-law distribution of simultaneous actions emerges, resulting in non-trivial aggregate fluctuations arising from micro-level interactions. In this model, the relevant configuration is the profile of relative prices. If a relatively large number of firms have their relative prices near the repricing threshold, a few small shocks cause many large avalanches of repricing behavior, leading to a quick decrease in the number of firms near the threshold. In contrast, if a relatively small number of firms are located near the threshold, only small-sized avalanches occur, and the number of firms near the threshold gradually rises. In either case, the relative price distribution converges toward the stationary distribution, at which the complementarity of repricing is $\theta$. Therefore, when $\theta$ is close to 1 , the relative prices globally converge to the point at which substantial fluctuations of the number of repricing firms emerge.

\subsection{Volatile short-run inflation under high long-run inflation}

Proposition 4 shows that the number of repricing firms exhibits higher volatility when the trend inflation is higher. In Proposition 2, we observed that the repricing size $q$ increases as the trend inflation increases. Therefore, both the extensive and intensive margins of aggregate price adjustments contribute to the higher volatility of inflation under higher trend inflation. This leads to the following main claim that the high inflation level causes high inflation variability.

Proposition 5. For sufficiently large $n$ and in a range of sufficiently large $\pi$, the variance of inflation $d \log P_{t}$ is increasing in $\pi$. 
The proof is provided in Appendix B.

In this section, we showed that the inflation rate fluctuates due to the complementarity of firms' repricing behavior under a finite number of firms $n$, and the volatility increases as the trend inflation level increases. To conclude this section, we discuss how this fluctuation sustains even when $n$ is very large. In conventional analysis of statedependent pricing, a continuum of firms is often assumed. As a direct consequence of the assumption, there is no aggregate fluctuation arising from idiosyncratic shocks. Such a setup is an innocuous abstraction of an economy with a large finite number of firms if the aggregate fluctuations arising from interaction exhibit a thin tail. For example, if the distribution of the number of firms that reprice simultaneously, $L$, has an exponential tail, then any moment is finite, and thus there is no aggregate fluctuations at an infinite limit of $n$. In the present model, this prediction holds for any finite $\pi$, since the distribution of $L$ is exponentially truncated. However, the truncation point is determined by $\theta$, and it could be deferred indefinitely by increasing $\pi$. At the limit $\theta=1$, the truncation point is deferred to infinity, and the distribution of $L$ becomes a pure power law. This can be seen in that the variance of $L$ is determined by an inverse of $1-\theta$. In this extreme case, there is no finite mean or variance of $L$, and the aggregate fluctuations survive even in an infinite limit of $n$. Thus, for any finite $n$, there is a finite level of $\pi$ with which the model economy exhibits sizable aggregate price fluctuations. In the next section, we investigate if the model economy exhibits meaningful fluctuations under realistic parameter calibration. 


\section{Numerical analysis}

In this section, we investigate the model quantitatively within a realistic range of longrun inflation rates under a finite number of firms. The purpose of this exercise is to extend the analytical results obtained asymptotically so far to the environment of low inflation rates. Thus, the quantification here should be regarded as a proof of concept rather than serious estimation of the model. We calibrate the model to match with some key empirical patterns reported by Nakamura and Steinsson (2008). They reported that the average size of price adjustment is about 7 percent. In the periods they studied (1988-2005), the U.S. monthly CPI experienced $0.1 \%$ inflation $(1.17 \%$ at the annual rate) and $0.23 \%$ standard deviations. Thus, we calibrate $\delta$ at 0.0023 so that $q$ is equal to 1.07 and calibrate $\mu$ at 0.1 so that the inflation volatility is $0.23 \%$, when the average annual inflation rate $\pi$ is set at $1.17 \%$. Note that $\mu$ is the arrival rate of exogenous repricing hazard. Since there are other firms that reprice by paying menu costs, the total fraction of repricing firms in the model is always greater than $\mu$. The elasticity of substitution $\eta$ is chosen to be 3 so that the labor share is two-thirds of the total value added. The time discount rate $\rho$ is set to the long-term real interest rate 0.02 .

We first show comparative statics results. The left panel of Figure 1 plots the stationary real wage $w$ on the left axis for each $\pi$. The plot confirms that the real wage is decreasing in $\pi$, extending our comparative statics (Proposition 2) to the low inflation range under the calibrated parameter set. The same plot shows the monthly repricing probability for a firm on the right axis, to quantify the comparative statics shown in Corollary 1. The right panel of Figure 1 plots the degree of complementarity $\theta$ and the standard deviation of the stochastic multiplier effect $\sqrt{\theta /(1-\theta)^{3}}$ for each $\pi$. In the plot, $\theta$ increases monotonically as $\pi$. This result extends the asymptotic 
analytical result of Proposition 3 to the range of low inflation rates. $\theta$ approaches to the critical value 1 quickly even in a low inflation range $2 \%-5 \%$. As a result, the stochastic multiplier effect increases almost linearly in $\pi$, as can be seen in the plot.
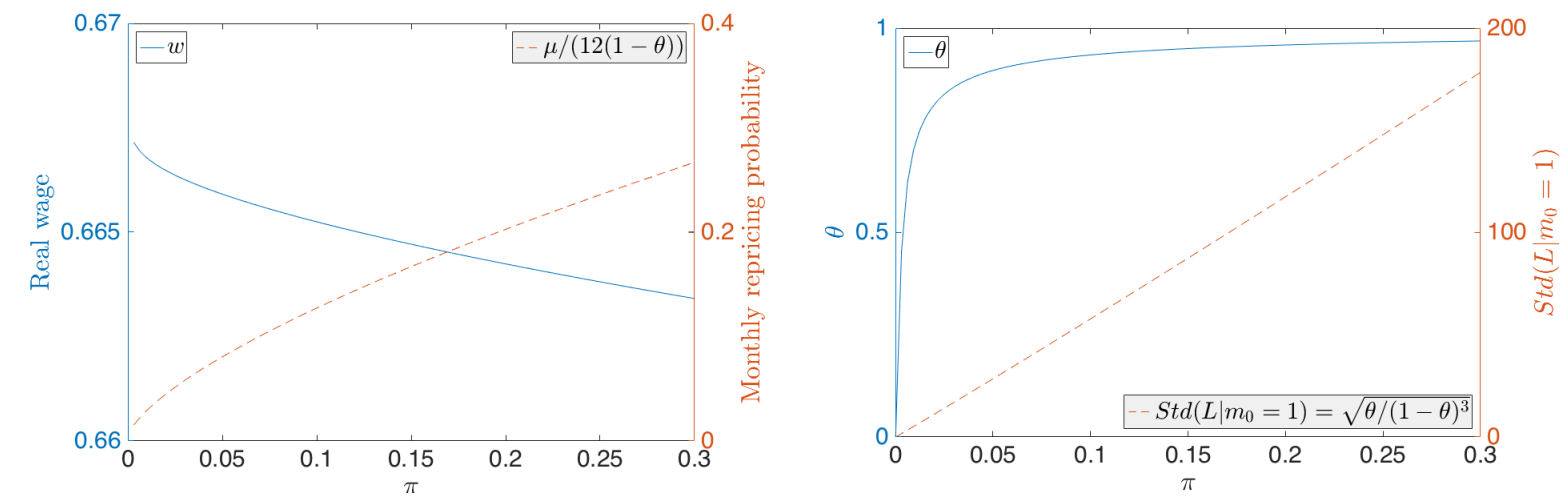

Figure 1: Comparative statics for various inflation level $\pi$. Left: Real wage rate (left axis) and repricing frequency (right axis) for various inflation levels $\pi$. Right: Degree of complementarity $\theta$ (left axis) and the standard deviation of $L$ conditional on $m_{0}=1$ (right axis).

The left panel of Figure 2 plots the stationary distribution $f(s)$ for each level of $\pi$. We observe that the exponential stationary distributions converge to the uniform distribution as $\pi$ increases. This implies that the increase in the degree of complementarity $\theta$ toward 1 along with $\pi$ is accompanied by the increase in $f_{o}$, the density of firms at the threshold $s=0$, toward 1 . The right panel of Figure 2 plots repricing size $q$ and threshold $\underline{p}$ for each $\pi$. Both numerical results show the quantitative extent of the analytical results obtained in Proposition 2.

Finally, Figure 3 plots the simulated standard deviation of monthly inflation rates for each $\pi$ under two cases $n=10000$ and $n=100000$. For each Monte Carlo trial, a state profile $\left(s_{1}, s_{2}, \ldots, s_{n}\right)$ is randomly drawn from a stationary joint density function 

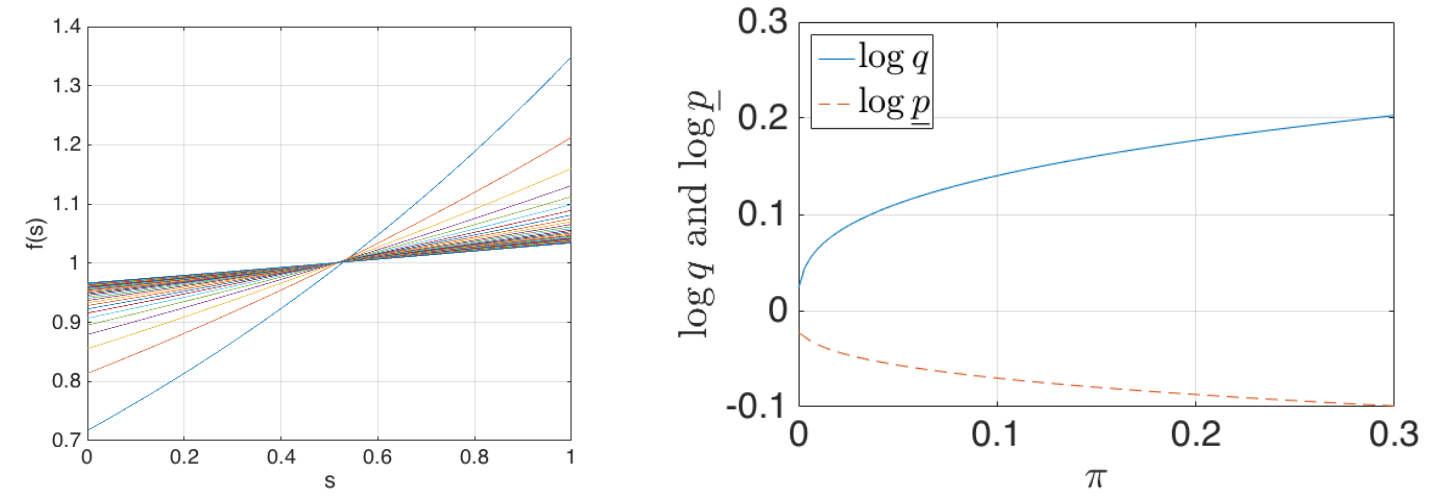

Figure 2: Left: Stationary distributions of $s$. The steepest slope (blue) corresponds to the inflation rate $\pi$ at $1 \%$, the second steepest (pink) at $2 \%$, and the flattest at $30 \%$. Right: Repricing size $q$ and repricing threshold relative price $\underline{p}$

$f^{n}(s)$. Next, a firm $i$ is selected randomly to receive a Calvo shock. Then, profile $s$ is updated as in Section 3, and the final profile $s$ and the number of firms $L$ that reprice following firm $i$ are computed. With these, the increase in aggregate price $d \log P$ is computed. This procedure is repeated for ten thousand times to compute the standard deviation of $d \log P$. The plotted result agrees with Proposition 5, for the standard deviation of inflation is increasing in the long-run inflation level $\pi$.

The simulated results are compared to the empirical observation between inflation level and volatility. Figure 4 plots the short-term volatility against the long-term level of inflation across countries or periods. A casual observation confirms the positive association between the level and volatility in a high inflation range, whereas this relationship seems somewhat attenuate in a low inflation range less than about $5 \%$ at annual rate. The dotted line in Figure 3 plots the same line as in the right panel of Figure 4 except that the standard deviation is reduced by the standard deviation 


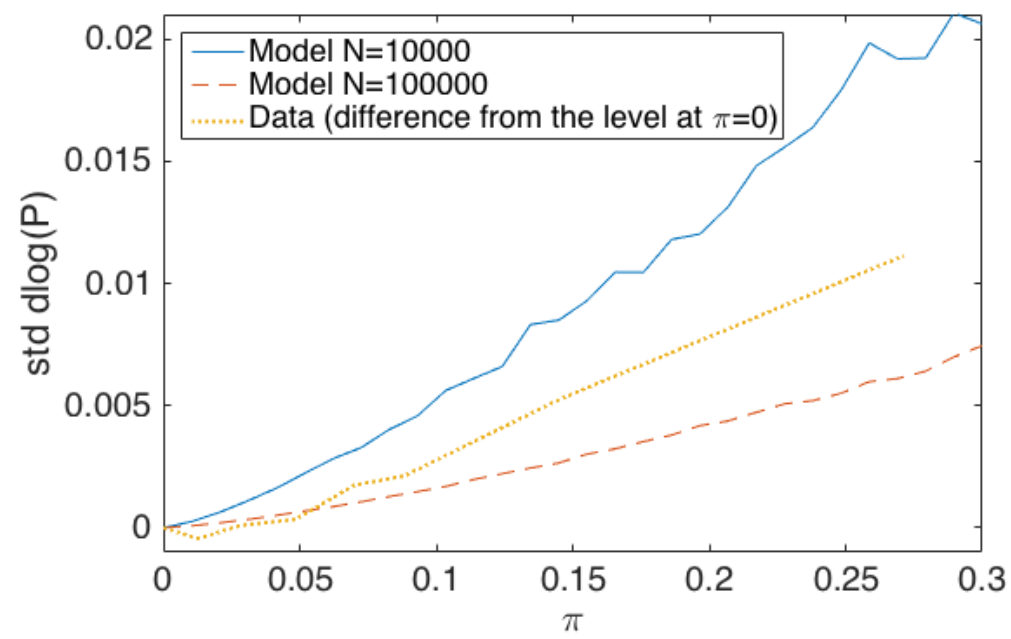

Figure 3: Levels of annual inflation rates and the standard deviations of monthly inflation rates in the model simulations and the data.

observed at $\pi=0$. Here, we interpret the standard deviation observed at $\pi=0$ as the price volatility caused by aggregate shocks which are not incorporated in the model. The plot shows that the observed relation is consistent with the case of $n$ between 10000 and 100000. This implies that the number of firms that affect a firm's pricing is a relevant parameter to determine the sensitivity of the aggregate price volatility to the inflation level.

The standard deviation of inflation rates is increased by more than 10 times for the increase of $\pi$ from $5 \%$ to $30 \%$ in Figure 3 (from $0.2 \%$ to $2 \%$ for $n=10000$; from $0.02 \%$ to $0.6 \%$ for $n=100000)$. This makes contrast with repricing size $\log q$ in the right panel of Figure 2, which increases less than twice (from 11\% to 20\%) when $\pi$ increases by the same degree. This observation implies that the extensive margin $(L)$ rather than the intensive margin $(\log q)$ accounts for the dominant portion of inflation 

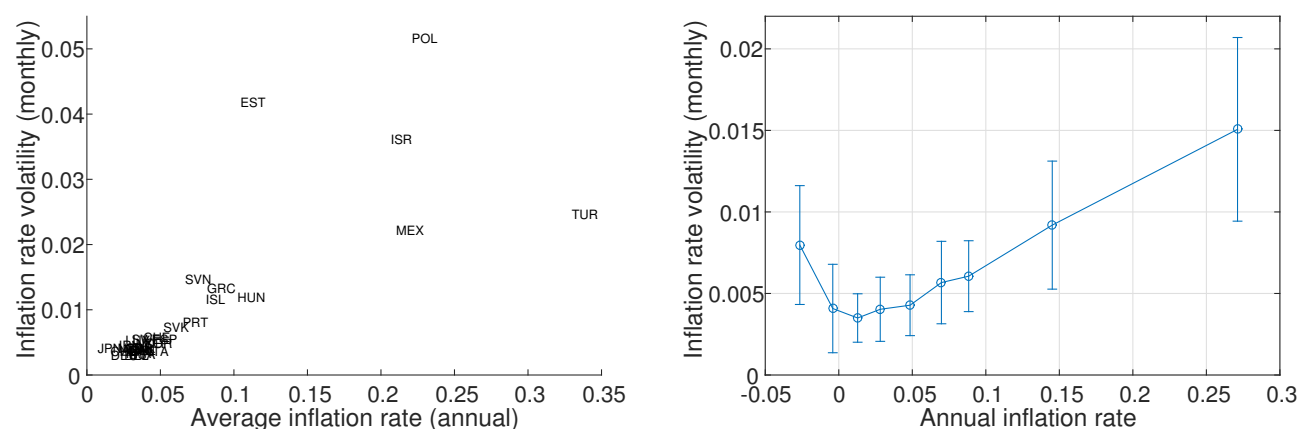

Figure 4: Inflation level and volatility. Left: Country-wise scatter plot of inflation level and volatility for 32 OECD countries for all the periods available in OECD database. The inflation rate is defined by a difference of logarithm of CPI. Right: Stratified pooled data. The horizontal axis shows annual inflation rates in two-year periods, while the vertical axis shows the volatility of monthly inflation rates during the two-year period. The error bar shows \pm 1 standard errors. The sample consists of 32 OECD countries during 1980-2015.

volatility for a high inflation range. In the model, the extensive margin causes the aggregate fluctuations, since without the complementarity of repricing behavior at the extensive margin there would be only negligible aggregate fluctuations. The numerical analysis shows that the extensive margin is important not only in the causal sense but also in quantitative terms for generating the positive association between the inflation level and volatility. 


\section{Conclusion}

This paper provides an explanation for the positive association between inflation level and inflation volatility. Since a firm's relative price is determined by other firms' pricing behavior, a firm's price increase necessarily leads to a decrease in other firms' relative prices. Thus, repricing behavior exhibits complementarity across firms. The complementarity leads to a possibility of stochastic synchronization of repricing behavior, in which the aggregate price exhibits fluctuations due to the varying number of firms that reprice simultaneously.

Analytical results demonstrate that the stochastic number of repricing firms exhibits a fat tail when the trend inflation is high. When the trend inflation is high, firms' relative prices are quickly drifted away from optimum, causing a relatively higher density of firms located at the repricing threshold. Hence, there is a higher probability for a firm's repricing to cause another firm's repricing. By this mechanism, we analytically show that a sufficiently high long-run inflation rate causes the high volatility in short-run inflation rates.

We calibrate the model with reasonable parameter values to investigate the levelvolatility nexus in a relatively low inflation range. Numerical analyses show that the considerable magnitude of inflation fluctuation and the positive association between level and volatility are generated by this mechanism. This suggests that there is another possible source of welfare costs of high inflation: a high inflation causes a high volatility in aggregate prices, leading to welfare loss due to intertemporal misallocation of resources. Further exploration of the welfare loss is left for future research.

\section{Appendix}




\section{A Proofs}

\section{Derivation of Equation (12)}

From the value matching condition $(8), z\left(p^{*}\right)=\rho v\left(p^{*}\right)$. Then, $v\left(p^{*}\right)-(\mu /(\rho+\mu)) v\left(p^{*}\right)=$

$z\left(p^{*}\right) /(\rho+\mu)$. Also, the smooth pasting condition $(10), z(\underline{p})=\rho v\left(p^{*}\right)-(\rho+\mu) \delta$, yields $z(\underline{p})=\rho v(\underline{p})-\mu \delta$. Using both equations, we have

$$
\begin{aligned}
v(\underline{p})-\frac{\mu}{\rho+\mu} v\left(p^{*}\right) & =\frac{z(\underline{p})+\mu \delta}{\rho}-\frac{z(\underline{p})+(\rho+\mu) \delta}{\rho} \frac{\mu}{\rho+\mu} \\
& =\frac{z(\underline{p})}{\rho+\mu} .
\end{aligned}
$$

Applying these to (7), we obtain, for both $p=p^{*}$ and $p=\underline{p}$,

$$
\frac{z(p)}{\rho+\mu}=c_{0} p^{-\frac{\rho+\mu}{\pi}}+\frac{p^{1-\eta}}{\rho+\mu-\pi(\eta-1)}-\frac{w p^{-\eta}}{\rho+\mu-\pi \eta} .
$$

By expanding the function $z(p)=p^{1-\eta}-w p^{-\eta}$, we have, for both $p=p^{*}$ and $p=p$,

$$
-\frac{c_{0}(\rho+\mu)}{\pi(\eta-1)} p^{-\frac{\rho+\mu}{\pi}}=\frac{p^{1-\eta}}{\rho+\mu-\pi(\eta-1)}-\frac{(\eta /(\eta-1)) w p^{-\eta}}{\rho+\mu-\pi \eta} .
$$

We substitute $c_{0}$ out by evaluating the above function at $p^{*}$ and $\underline{p}$ to obtain

$$
q^{-\frac{\rho+\mu}{\pi}}=\frac{p^{* 1-\eta}-c_{1} w p^{*-\eta}}{\underline{p}^{1-\eta}-c_{1} w \underline{p}^{-\eta}}
$$

where

$$
c_{1} \equiv \frac{\eta}{\eta-1} \frac{\eta-1-\frac{\rho+\mu}{\pi}}{\eta-\frac{\rho+\mu}{\pi}} .
$$

By using $p^{*}=q \underline{p}$ and rearranging, we obtain (12) as follows.

$$
\begin{aligned}
& q^{-\frac{\rho+\mu}{\pi}}\left(\underline{p}-c_{1} w\right) \underline{p}^{-\eta}=\left(q^{1-\eta} \underline{p}-c_{1} w q^{-\eta}\right) \underline{p}^{-\eta} \\
\Leftrightarrow & \underline{p}\left(q^{\eta-1-\frac{\rho+\mu}{\pi}}-1\right)=c_{1} w\left(q^{\eta-1-\frac{\rho+\mu}{\pi}}-q^{-1}\right) \\
\Leftrightarrow & \underline{p} \varphi(q, \eta-1-(\rho+\mu) / \pi)=\frac{\eta}{\eta-1} \frac{w}{q} \varphi(q, \eta-(\rho+\mu) / \pi)
\end{aligned}
$$




\section{Properties of $\varphi(q, x)$}

We summarize some properties of the function $\varphi(q, x)$ in the following lemma. Denote the first derivatives as $\varphi_{q}(q, x) \equiv(\partial / \partial q) \varphi(q, x)$ and $\varphi_{x}(q, x) \equiv(\partial / \partial x) \varphi(q, x)$.

Lemma 1. For $q>1, \varphi(q, x) \equiv\left(q^{x}-1\right) / x$ satisfies the following properties.

(i) $\varphi(q, x)>0, \varphi_{q}(q, x)>0$, and $\varphi(q,-x)=\varphi(q, x) / q^{x}=\varphi(q, x) /\left(\varphi_{q}(q, x) q\right)$

(ii) $\log \varphi(q, x)$ is strictly increasing and convex in $x$ :

$$
\begin{aligned}
\frac{\varphi_{x}}{\varphi}(q, x) & =\frac{\log q}{1-q^{-x}}-\frac{1}{x}>0 \\
\frac{\partial}{\partial x} \frac{\varphi_{x}}{\varphi}(q, x) & =\frac{1}{x^{2}}\left(1-\frac{q^{x}\left(\log q^{x}\right)^{2}}{\left(q^{x}-1\right)^{2}}\right) \geq 0 \quad \text { with equality holding at } x=0
\end{aligned}
$$

(iii) $(\partial / \partial q)(\varphi(q, x) / \varphi(q, y))>0$ if and only if $x>y$

(iv) $(\partial / \partial q)\left(q^{x} \varphi(q, y) / \varphi(q, x+y)\right)>0$ if and only if $x>0$

(v) $(\partial / \partial q)(\varphi(q, x) \varphi(q, y) / \varphi(q, x+y))>0$ if $x>0$

(vi) A derivative

$$
\frac{\varphi_{x}}{\varphi^{2}}(q, x)=\frac{\partial}{\partial x} \frac{-1}{\varphi(q, x)}=\frac{q^{x} \log \left(q^{x}\right)-q^{x}+1}{\left(q^{x}-1\right)^{2}}>0
$$

is decreasing in $x$.

Proof:

(i) $\varphi(q, x)=\left(q^{x}-1\right) / x>0$ for $q>1$ and $x \neq 0$, and $\lim _{x \rightarrow 0} \varphi(q, x)=\log q>0$ for $q>1$. Also, $\varphi_{q}(q, x)=q^{x-1}>0$. Furthermore, $\varphi(q,-x) q^{x}=\left(1-q^{x}\right) /(-x)=\varphi(q, x)$. 
(ii) By taking a partial derivative, we obtain

$$
\varphi_{x}(q, x)=\frac{\partial}{\partial x} \frac{q^{x}-1}{x}=\frac{1}{x^{2}}\left[q^{x} \log \left(q^{x}\right)-\left(q^{x}-1\right)\right] .
$$

A function $y \log y-(y-1)$ is strictly positive for $y>0$ and $y \neq 1$. Hence, $\varphi_{x}(q, x)$ is strictly positive for $x \neq 0$. For $x=0$, L'Hopital's rule yields $\lim _{x \rightarrow 0} \varphi_{x}(q, x)=$ $(\log q)^{2} / 2$. Hence, $\varphi_{x}(q, x)>0$ for any $x$. Moreover,

$$
\frac{\varphi_{x}}{\varphi}(q, x)=\frac{\partial}{\partial x} \log \frac{q^{x}-1}{x}=\frac{x}{q^{x}-1} \frac{\left(q^{x} \log q\right) x-\left(q^{x}-1\right)}{x^{2}}=\frac{\log q}{1-q^{-x}}-\frac{1}{x}
$$

and

$$
\frac{\partial}{\partial x} \frac{\varphi_{x}}{\varphi}(q, x)=-\frac{q^{-x}(\log q)^{2}}{\left(1-q^{-x}\right)^{2}}+\frac{1}{x^{2}}=\frac{1}{x^{2}}\left(1-\frac{q^{x}\left(\log q^{x}\right)^{2}}{\left(q^{x}-1\right)^{2}}\right) .
$$

Now consider a function $h(y)=(y-1)^{2}-y(\log y)^{2}$ for $y>0$. Note that $h^{\prime}(y)=$ $2(y-1)-2 \log y-(\log y)^{2}$ and $h(1)=h^{\prime}(1)=0$. Also, $h^{\prime \prime}(y)=(2 / y)(y-1-\log y)$ is positive for $y>0$. Thus, $h^{\prime}$ is increasing in $y$, implying that $h^{\prime}(y)<0$ for $0<y<1$ and $h^{\prime}(y)>0$ for $y>1$. Hence, $h(y)$ takes minimum 0 at $y=1$ and $h(y)>0$ for $y \neq 1$. This leads to $1 \geq y(\log y)^{2} /(y-1)^{2}$ for $y>0$ with equality holding at $y=1$. Thus, we obtain $\left(\partial^{2} / \partial x^{2}\right) \log \varphi(q, x) \geq 0$ with equality holding at $x=0$.

(iii) By direct calculation, we obtain for the case $x \neq 0$ and $y \neq 0$ :

$$
\begin{aligned}
\frac{\partial}{\partial q} \frac{y}{x} \frac{q^{x}-1}{q^{y}-1} & =\frac{y}{x\left(q^{y}-1\right)^{2}}\left(x q^{x-1}\left(q^{y}-1\right)-y q^{y-1}\left(q^{x}-1\right)\right) \\
& =\frac{y^{2} q^{x+y-1}}{\left(q^{y}-1\right)^{2}}\left(\frac{1-q^{-y}}{y}-\frac{1-q^{-x}}{x}\right) \\
& =\frac{q^{x+y-1}}{\varphi^{2}(q, y)}(\varphi(q,-y)-\varphi(q,-x)) .
\end{aligned}
$$

It is verified that the above expression also holds for the case $x=0$ or $y=0$. By Lemma 1(ii), $\varphi(q, x)$ is increasing in $x$. Hence, the last expression has the same sign as $x-y$. 
(iv) We obtain for $y \neq 0$ :

$$
\begin{aligned}
& \frac{\partial}{\partial q} \frac{q^{x} \varphi(q, y)}{\varphi(q, x+y)}=\frac{\partial}{\partial q} \frac{q^{x}\left(q^{y}-1\right)}{q^{x+y}-1} \frac{x+y}{y} \\
& =\frac{x+y}{y\left(q^{x+y}-1\right)^{2}}\left[\left(q^{x+y}-1\right)\left((x+y) q^{x+y-1}-x q^{x-1}\right)-(x+y) q^{x+y-1}\left(q^{x+y}-q^{x}\right)\right] \\
& =\frac{x+y}{y\left(q^{x+y}-1\right)^{2}}\left(y q^{2 x+y-1}-(x+y) q^{x+y-1}+x q^{x-1}\right) \\
& =\frac{x(x+y) q^{x+y-1}}{\left(q^{x+y}-1\right)^{2}}\left(\frac{q^{x}-1}{x}-\frac{q^{-y}-1}{-y}\right) \\
& =x\left[\frac{(x+y) q^{x+y-1}}{\left(q^{x+y}-1\right)^{2}}(\varphi(q, x)-\varphi(q,-y))\right] .
\end{aligned}
$$

The same expression holds for the case $y=0$. Using Lemma 1 (ii) stating that $\varphi(q, x)$ is increasing in $x$, the term inside the square brackets has the same sign as $x+y$. Since there is another $x+y$ term, the expression inside the square brackets is positive. Hence, the entire right-hand side expression has the same sign as $x$.

(v) We consider the derivative of

$$
\frac{\varphi(q, x) \varphi(q, y)}{\varphi(q, x+y)}=\frac{q^{x} \varphi(q, y)}{x \varphi(q, x+y)}-\frac{\varphi(q, y)}{x \varphi(q, x+y)}
$$

Since $x>0$, the first term is strictly increasing in $q$ by (iv). Moreover, the second term (including the negative sign) is strictly increasing in $q$ by (iii). Therefore the entire expression is increasing in $q$.

(vi) Define a function $g(y)=(y \log y-y+1) /(y-1)^{2}$. Note that $y \log y>y-1$ for $y \neq 1$. Also, by L'Hopital's rule, we have

$$
g(1)=\lim _{y \rightarrow 1} \frac{\log y}{2(y-1)}=\lim _{y \rightarrow 1} \frac{1}{2 y}=1 / 2 .
$$


Thus, $g(y)$ is continuous and $g(y)>0$. Moreover,

$$
\begin{aligned}
g^{\prime}(y) & =\frac{1}{(y-1)^{4}}\left((y-1)^{2} \log y-2(y-1)(y \log y-y+1)\right) \\
& =\frac{1}{(y-1)^{3}}(2(y-1)-(y+1) \log y) .
\end{aligned}
$$

Note that a function $h(y) \equiv 2(y-1)-(y+1) \log y$ satisfies $h(1)=0$ and

$$
h^{\prime}(y)=1-1 / y-\log y \leq 0 \quad \text { with equality holding at } y=1
$$

This implies that $h(y)$ is positive for $0<y<1$ and negative for $y>1$. Hence, $g^{\prime}(y)=h(y) /(y-1)^{3}$ is negative for both $0<1<y$ and $y>1$. This implies that $g(y)$ is a decreasing function for $y>0$. Hence, $(\partial / \partial x)(-1 / \varphi(q, x))=g\left(q^{x}\right)$ is a decreasing function in $x$ for $q>1$.

\section{Proof of Proposition 1: There exists a unique steady state equilibrium for} each $\pi>0$.

Let $\delta_{o}, A$, and $B$ denote $\delta_{o} \equiv \delta(\rho+\mu) /(\eta-1)$ and

$$
\begin{aligned}
A & \equiv \frac{\varphi(q,-\eta)}{\varphi(q, 1-\eta)} \frac{\varphi\left(q,-\eta+1+\frac{\rho+\mu}{\pi}\right)}{\varphi\left(q,-\eta+\frac{\rho+\mu}{\pi}\right)} \\
B & \equiv \frac{\varphi(q, 1-\eta+\mu / \pi)}{\varphi(q, \mu / \pi) \varphi(q, 1-\eta)}
\end{aligned}
$$

to write Equation (15) as

$$
A=B \delta_{o}+1
$$

$B$ is decreasing in $q$ by Lemma $1(\mathrm{v})$. Since any ratio $\varphi(q, x) / \varphi(q, y)$ converges to 1 as $q \rightarrow 1$ by L'Hopital's rule, and since $\lim _{q \rightarrow 1} \varphi(q, \mu / \pi)=0, B$ tends to $\infty$ as $q \rightarrow 1$ for any $\pi$. Also, $B$ converges to 0 as $q \rightarrow \infty$, because for any $x>0, \lim _{q \rightarrow \infty} \varphi(q, x)=$ $\infty$ and $\lim _{q \rightarrow \infty} \varphi(q,-x)=1 / x$. Thus, the right-hand side of (23) is a continuously decreasing function for $q>1$, travelling from $\infty$ to 1 . 
Next we investigate the left-hand side of (23). We use notation $a \equiv(\rho+\mu) / \pi$. A takes value 1 at $q=1$. Using Lemma 1(i), we obtain the relation

$$
A=\frac{\varphi(q,-\eta)}{\varphi(q, 1-\eta)} \frac{\varphi(q,-\eta+1+a)}{\varphi(q,-\eta+a)}=\frac{\varphi(q, \eta)}{\varphi(q, \eta-1)} \frac{\varphi(q, \eta-1-a)}{\varphi(q, \eta-a)} .
$$

Its log-derivative with respect to $q$ is

$$
\begin{aligned}
& A_{q} / A \equiv \frac{\partial}{\partial q} \log A \\
& =\frac{\partial}{\partial q}(\log \varphi(q, \eta)-\log \varphi(q, \eta-1)+\log \varphi(q, \eta-1-a)-\log \varphi(q, \eta-a)) \\
& =\frac{1}{q}\left(\frac{q^{\eta}}{\varphi(q, \eta)}-\frac{q^{\eta-1}}{\varphi(q, \eta-1)}+\frac{q^{\eta-1-a}}{\varphi(q, \eta-1-a)}-\frac{q^{\eta-a}}{\varphi(q, \eta-a)}\right) \\
& =\frac{1}{q}\left(\frac{1}{\varphi(q,-\eta)}-\frac{1}{\varphi(q, 1-\eta)}+\frac{1}{\varphi(q, 1+a-\eta)}-\frac{1}{\varphi(q, a-\eta)}\right) \\
& =\frac{1}{q}\left(\int_{-\eta}^{\min (1-\eta, a-\eta)} \frac{\partial}{\partial x} \frac{-1}{\varphi(q, x)} d x-\int_{\max (1-\eta, a-\eta)}^{1+a-\eta} \frac{\partial}{\partial x} \frac{-1}{\varphi(q, x)} d x\right) .
\end{aligned}
$$

By Lemma 1(vi), the integrand $(\partial / \partial x)(-1 / \varphi(q, x))$ in $(25)$ is a positive-valued, decreasing function. This implies that $A_{q}$ is strictly positive, since $-\eta<\min (1-\eta, a-$ $\eta) \leq \max (1-\eta, a-\eta)<1+a-\eta$. Hence, the left-hand side of (23) is increasing in $q$ and takes values above 1 . Hence, Equation (23) has a unique solution $q$ in the range $q>1$.

\section{Proof of Proposition 2:}

1. Repricing size $\log q$ increases unboundedly as $\pi$ increases.

2. Markup $p^{*} / w$ increases as $\pi$ increases.

3. Real wage $w$ decreases as $\pi$ increases for sufficiently large $\pi$. 
We will establish that $q$ is increasing in $\pi$ in (23). By taking total derivative of (23) and rearranging, we have

$$
\frac{d \log q}{d \pi}=\frac{-1}{q} \frac{A_{\pi}-\delta_{o} B_{\pi}}{A_{q}-\delta_{o} B_{q}} .
$$

$A_{q}$ was shown positive in the proof of the last proposition. $B_{q}$ is negative by Lemma $1(\mathrm{v})$. Hence, the denominator $q\left(A_{q}-\delta_{o} B_{q}\right)$ is positive.

Using the right-hand side of (24), we calculate $A_{\pi}$ as

$$
\begin{aligned}
\frac{A_{\pi}}{A} & =\frac{\partial \log A}{\partial \pi}=\left(-\frac{\varphi_{x}}{\varphi}(q, \eta-1-a)+\frac{\varphi_{x}}{\varphi}(q, \eta-a)\right) \frac{-a}{\pi} \\
& =\int_{\eta-1-a}^{\eta-a} \frac{1}{x^{2}}\left(1-\frac{q^{x}\left(\log q^{x}\right)^{2}}{\left(q^{x}-1\right)^{2}}\right) d x\left(\frac{-a}{\pi}\right)
\end{aligned}
$$

where the second line used Lemma 1(ii). Since the integrand is positive by Lemma 1(ii), we obtain $0>A_{\pi}>-(a / \pi) /(\eta-1-a)^{2}$. Similarly, $B_{\pi}$ is calculated as

$$
B_{\pi}=\frac{\partial \log B}{\partial \pi} B=\left(\frac{\varphi_{x}}{\varphi}(1-\eta+\mu / \pi)-\frac{\varphi_{x}}{\varphi}(\mu / \pi)\right)\left(\frac{-\mu}{\pi^{2}}\right) \frac{\varphi(q, 1-\eta+\mu / \pi)}{\varphi(q, \mu / \pi) \varphi(q, 1-\eta)} .
$$

By Lemma 1(ii), $\log \varphi(q, x)$ is convex in $x$. Thus, the first bracket is negative, implying that $B_{\pi}$ is positive. Combining with the previous result on $A_{\pi}$, we obtain that the numerator $-A_{\pi}+\delta_{o} B_{\pi}$ is positive. This establishes that $d \log q / d \pi>0$.

Next, we show that $\log q$ increases indefinitely as $\pi \rightarrow \infty$. For any fixed $q, A$ converges to 1 as $\pi \rightarrow \infty$. Hence, $B$ converges to 0 . The numerator of $B, \varphi(q, 1-\eta+\mu / \pi)$, is strictly positive and increasing in $q$ for $q>1$. Therefore, the denominator must tend to infinity in order for $B$ to vanish to 0 . However, $\varphi(q, 1-\eta)$ is bounded. Thus, $\varphi(q, \mu / \pi)$ must tend to infinity. Now suppose that $\log q$ is bounded above when $\pi$ tends to infinity. Then $\lim _{\pi \rightarrow \infty}\left(e^{\mu(\log q) / \pi}-1\right) /(\mu / \pi)=\lim _{\pi \rightarrow \infty}\left(e^{\mu(\log q) / \pi} \mu(\log q) /\left(-\pi^{2}\right)\right) /\left(\mu /\left(-\pi^{2}\right)\right)=$ $\lim _{\pi \rightarrow \infty} \log q<\infty$. This contradicts with that $\varphi(q, \mu / \pi)$ diverges. Hence, $\log q$ must diverge toward positive infinity as $\pi \rightarrow \infty$. 
From (12) we obtain

$$
\frac{p^{*}}{w} \frac{\eta-1}{\eta}=\frac{\varphi\left(q, \eta-\frac{\rho+\mu}{\pi}\right)}{\varphi\left(q, \eta-1-\frac{\rho+\mu}{\pi}\right)} .
$$

The right-hand side is increasing in $q$ by Lemma 1(iii) and increasing in $\pi$ by Lemma 1(ii).

Finally, real wage $w$ is pinned down using $(14,12)$ as

$$
\begin{aligned}
w & =\frac{\eta-1}{\eta} \frac{q \varphi\left(q, \eta-1-\frac{\rho+\mu}{\pi}\right)}{\varphi\left(q, \eta-\frac{\rho+\mu}{\pi}\right)}\left(\frac{\varphi(q, 1-\eta+\mu / \pi)}{\varphi(q, \mu / \pi)}\right)^{1 /(\eta-1)} \\
& =\frac{\eta-1}{\eta} \frac{\varphi\left(q, 1-\eta+\frac{\rho+\mu}{\pi}\right)}{\varphi\left(q,-\eta+\frac{\rho+\mu}{\pi}\right)}\left(\frac{\varphi(q, 1-\eta+\mu / \pi)}{\varphi(q, \mu / \pi)}\right)^{1 /(\eta-1)} .
\end{aligned}
$$

Note that $\varphi(q, \mu / \pi)$ tends to infinity as $\pi \rightarrow \infty$ and all the other $\varphi$ functions above are finitely bounded for $q>1$. Thus, for sufficiently large $\pi, w$ decreases as $\pi$ increases. This competes the proof.

Asymptotic behavior of $d \log q / d \pi$ :

\section{Lemma 2.}

$$
\frac{d \log q}{d \pi} \sim \frac{\mu}{x_{1}^{2} \delta_{o}} \frac{\varphi(q, \mu / \pi) \varphi(q,-\mu / \pi)}{\pi^{2}}
$$

where $x_{1} \in[\eta-1-a, \eta-a]$. 
Proof: We start from (25).

$$
\begin{aligned}
A_{q} & =\frac{A}{q}\left(\int_{-\eta}^{\min (1-\eta, a-\eta)} \frac{\partial}{\partial x} \frac{-1}{\varphi(q, x)} d x-\int_{\max (1-\eta, a-\eta)}^{1+a-\eta} \frac{\partial}{\partial x} \frac{-1}{\varphi(q, x)} d x\right) \\
& =\frac{A a}{q}\left[\frac{\partial}{\partial x} \frac{-1}{\varphi}\left(q, x_{2}\right)-\frac{\partial}{\partial x} \frac{-1}{\varphi}\left(q, x_{3}\right)\right] \quad \text { where } x_{2} \in[-\eta, a-\eta] \text { and } x_{3} \in[1-\eta, 1+a-\eta] \\
& =\frac{A a}{q} \int_{x_{3}}^{x_{2}} \frac{\partial^{2}}{\partial x^{2}} \frac{-1}{\varphi}(q, x) d x \\
& \sim \frac{A a}{q} \int_{1-\eta}^{-\eta} \frac{\partial^{2}}{\partial x^{2}} \frac{-1}{\varphi}(q, x) d x \\
& =\frac{A a}{q}\left[-\frac{2 q^{x_{4}} \log q}{\left(q^{x_{4}}-1\right)^{2}}\left(1-\frac{q^{x_{4}}+1}{q^{x_{4}}-1} \frac{\log q^{x_{4}}}{2}\right)\right] \quad \text { for some } x_{4} \in[-\eta, 1-\eta] \\
& \sim-x_{4} a q^{x_{4}-1}(\log q)^{2}>0,
\end{aligned}
$$

where $x(t) \sim y(t)$ denotes the relation $\lim _{t \rightarrow \infty} x(t) / y(t)=1$. Moreover, we obtain the following asymptotic results by collecting the leading terms of $(26,27)$ respectively:

$$
\begin{aligned}
A_{\pi} & \sim-\frac{a}{\pi} \frac{1}{x_{1}^{2}}, \quad \text { for some } x_{1} \in[\eta-1-a, \eta-a], \\
B_{\pi} & =\left(\frac{\varphi_{x}}{\varphi}(q, 1-\eta+\mu / \pi)-\frac{\varphi_{x}}{\varphi}(q, \mu / \pi)\right)\left(\frac{-\mu}{\pi^{2}}\right) \frac{\varphi(q, 1-\eta+\mu / \pi)}{\varphi(q, \mu / \pi) \varphi(q, 1-\eta)} \\
& \sim\left(\frac{\log q}{-q^{\eta-1-\mu / \pi}}+\frac{1}{\eta-1-\mu / \pi}-\frac{\log q}{1-q^{-\mu / \pi}}+\frac{\pi}{\mu}\right)\left(\frac{-\mu}{\pi^{2}}\right) \frac{(\eta-1) /(\eta-1-\mu / \pi)}{\varphi(q, \mu / \pi)} \\
& \sim\left(\frac{1}{\pi(\eta-1)-\mu}-\frac{\log q}{\mu \varphi(q,-\mu / \pi)}+\frac{1}{\mu}\right) \frac{-\mu(\eta-1) /(\eta-1-\mu / \pi)}{\pi \varphi(q, \mu / \pi)} \\
& \sim\left(\frac{\log q}{\varphi(q,-\mu / \pi)}-1\right) \frac{(\eta-1) /(\eta-1-\mu / \pi)}{\pi \varphi(q, \mu / \pi)} .
\end{aligned}
$$


We also obtain

$$
\begin{aligned}
-B_{q} & =-\frac{\partial \log B}{\partial q} B \\
& =\left(\frac{1}{\varphi(q,-\mu / \pi)}+\frac{1}{\varphi(q, \eta-1)}-\frac{1}{\varphi(q, \eta-1-\mu / \pi)}\right) \frac{B}{q} \\
& =\left(\frac{1}{\varphi(q,-\mu / \pi)}+\int_{\eta-1-\mu / \pi}^{\eta-1} \frac{\partial}{\partial x} \frac{1}{\varphi}(q, x) d x\right) \frac{B}{q} \\
& =\left(\frac{1}{\varphi(q,-\mu / \pi)}-\frac{\mu}{\pi} \frac{\varphi_{x}}{\varphi^{2}}\left(q, x_{5}\right)\right) \frac{B}{q} \text { where } x_{5} \in[\eta-1-\mu / \pi, \eta-1] \\
& =\left(\frac{1}{\varphi(q,-\mu / \pi)}-\frac{\mu}{\pi} \frac{\varphi_{x}}{\varphi^{2}}\left(q, x_{5}\right)\right) \frac{\varphi(q, 1-\eta+\mu / \pi)}{\varphi(q, \mu / \pi) \varphi(q, 1-\eta) q} \\
& \sim \frac{1}{\varphi(q, \mu / \pi) \varphi(q,-\mu / \pi) q}
\end{aligned}
$$

where we used that $\left(\varphi_{x} / \varphi^{2}\right)\left(q, x_{5}\right)$ is dominated by $1 / \varphi(q,-\mu / \pi)$ for $x_{5}>0$.

With these, we will evaluate

$$
\frac{d \log q}{d \pi}=\frac{-1}{q} \frac{A_{\pi}-\delta_{o} B_{\pi}}{A_{q}-\delta_{o} B_{q}}
$$

The numerator is

$$
\begin{aligned}
-A_{\pi}+\delta_{o} B_{\pi} & \sim \frac{a}{\pi x_{1}^{2}}+\delta_{o}\left(\frac{\log q}{\varphi(q,-\mu / \pi)}-1\right) \frac{(\eta-1) /(\eta-1-\mu / \pi)}{\pi \varphi(q, \mu / \pi)} \\
& =\frac{a}{\pi x_{1}^{2}}+\frac{\delta_{o}}{\varphi(q,-\mu / \pi)}\left(\int_{-\mu / \pi}^{0} q^{x-1} d x\right) \frac{(\eta-1) /(\eta-1-\mu / \pi)}{\pi \varphi(q, \mu / \pi)} \\
& \sim \frac{\mu}{\pi^{2} x_{1}^{2}}+\frac{\delta_{o} q^{x_{6}-1}(\mu / \pi)}{\varphi(q,-\mu / \pi) \varphi(q, \mu / \pi) \pi} \quad \text { where } x_{6} \in[-\mu / \pi, 0] \\
& \sim \frac{\mu}{x_{1}^{2} \pi^{2}} .
\end{aligned}
$$

Using $x_{4}<0$, the denominator is

$$
\begin{aligned}
q\left(A_{q}-\delta_{o} B_{q}\right) & \sim-\frac{x_{4} \mu}{\pi} q^{x_{4}}(\log q)^{2}+\frac{\delta_{o}}{\varphi(q, \mu / \pi) \varphi(q,-\mu / \pi)} \\
& \sim \frac{\delta_{o}}{\varphi(q, \mu / \pi) \varphi(q,-\mu / \pi)} .
\end{aligned}
$$


Thus,

$$
\frac{d \log q}{d \pi} \sim \frac{\mu}{x_{1}^{2} \delta_{o}} \frac{\varphi(q, \mu / \pi) \varphi(q,-\mu / \pi)}{\pi^{2}}
$$

where $x_{1} \in[\eta-1-a, \eta-a]$.

Proof of Proposition 3: Degree of complementarity $\theta$ is increasing in $\pi$ for sufficiently large $\pi$.

A firm at the threshold $s_{i, t}=0$ reprices its price by $\Delta \log P_{i, t}=\log q$. Substituting this into (16), its impact on $P_{t}$ is written as $n \Delta \log P_{t}=\underline{p}_{i, t}^{1-\eta} \varphi(q, 1-\eta)+O\left(n^{-1}\right)$. Using (13) and (14), we obtain

$$
\begin{aligned}
\theta & =f_{o} \varphi(q, 1-\eta) \underline{p}^{1-\eta} / \log q \\
& =\frac{\log q}{\varphi(q, \mu / \pi)} \varphi(q, 1-\eta) \frac{\varphi(q, \mu / \pi)}{\varphi(q, 1-\eta+\mu / \pi)} \frac{1}{\log q} \\
& =\frac{\varphi(q, 1-\eta)}{\varphi(q, 1-\eta+\mu / \pi)} .
\end{aligned}
$$

Note that $\theta<1$.

By taking total derivative of $\theta$, we obtain

$$
\frac{d \log \theta}{d \pi}=\left(\frac{1}{\varphi(q, \eta-1)}-\frac{1}{\varphi(q, \eta-1-\mu / \pi)}\right) \frac{d \log q}{d \pi}+\frac{\varphi_{x}}{\varphi}(q, 1-\eta+\mu / \pi) \frac{\mu}{\pi^{2}} .
$$

The second term is

$$
\left(\frac{\log q}{1-q^{\eta-1-\mu / \pi}}+\frac{1}{\eta-1-\mu / \pi}\right) \frac{\mu}{\pi^{2}} .
$$

$\log q /\left(1-q^{\eta-1-\mu / \pi}\right)$ converges to 0 as $\pi$ and $q$ increase indefinitely. Thus, the second term in (29) asymptotes to $(\mu /(\eta-1)) \pi^{-2}$. 
The first term is

$$
\begin{aligned}
\left(\frac{1}{\varphi(q, \eta-1)}-\frac{1}{\varphi(q, \eta-1-\mu / \pi)}\right) \frac{d \log q}{d \pi} & =-\int_{\eta-1-\mu / \pi}^{\eta-1} \frac{\partial}{\partial x} \frac{-1}{\varphi}(q, x) d x \frac{d \log q}{d \pi} \\
& >-\frac{\partial}{\partial x} \frac{-1}{\varphi}\left(q, x_{7}\right) \frac{\mu}{\pi} \frac{\overline{d \log q}}{d \pi} \\
& =-\frac{q^{x_{7}} \log q^{x_{7}}-\left(q^{x_{7}}-1\right)}{\left(q^{x_{7}}-1\right)^{2}} \frac{\mu}{\pi} \frac{\overline{d \log q}}{d \pi} \\
& \sim-\frac{x_{7} \log q}{q^{x_{7}}} \frac{\mu}{\pi} \frac{\mu}{x_{1}^{2} \delta_{o}} \frac{(\log q)^{2}}{\pi^{2} q^{-\mu / \pi}} \\
& =-\frac{x_{7}(\log q)^{3}}{q^{\eta-1-2 \mu / \pi}} \frac{\mu^{2}}{x_{1}^{2} \delta_{o} \pi^{3}}
\end{aligned}
$$

where $x_{7} \in[\eta-1-\mu / \pi, \eta-1]$. Thus, this negative first term of (29) is dominated by the positive second term for $\eta-1-2 \mu / \pi>0$. Hence, we obtain that $d \log \theta / d \pi>0$ for sufficiently large $\pi$.

Proof of Proposition 4: The asymptotic variance of $L$ as $n \rightarrow \infty$ conditional on $m_{0}=1$ is $\theta /(1-\theta)^{3}$. Furthermore, as $n \rightarrow \infty$, the distribution of $L$ conditional on $m_{0}$ asymptotically follows:

$$
\begin{aligned}
\operatorname{Pr}\left(L=\ell \mid m_{0}\right) & =\frac{m_{0}}{\ell} \frac{e^{-\theta \ell}(\theta \ell)^{\ell-m_{0}}}{\left(\ell-m_{0}\right) !} \\
& \propto e^{-(\theta-1-\log \theta) \ell} \ell^{-1.5} \quad \text { as } \ell \rightarrow \infty .
\end{aligned}
$$

The probability generating function $\Psi(z)$ of the sum $m_{1}$ of a branching process with initial value 1 has a recursive form as $\Psi(z)=z \Phi(\Psi(z))$, where $\Phi$ is a probability generating function of the number of children born from a parent. In our case, $\Phi$ follows a Poisson distribution with mean $\theta$ asymptotically as $n \rightarrow \infty$. By the property of a probability generating function, $\Phi(1)=\Psi(1)=1$. Moreover, $\Phi^{\prime}(1)$ is equal to 
the mean and $\Phi^{\prime \prime}(1)+\Phi^{\prime}(1)-\left(\Phi^{\prime}(1)\right)^{2}$ is equal to the variance. Thus, $\Phi^{\prime}(1)=\theta$ and $\Phi^{\prime \prime}(1)+\Phi^{\prime}(1)-\left(\Phi^{\prime}(1)\right)^{2}=\theta$. Hence, $\Phi^{\prime \prime}(1)=\theta^{2}$.

Using the recursive relationship, we obtain $\Psi^{\prime}(z)=\Phi(\Psi(z))+z \Phi^{\prime}(\Psi(z)) \Psi^{\prime}(z)$ and $\Psi^{\prime \prime}(z)=2 \Phi^{\prime}(\Psi(z)) \Psi^{\prime}(z)+z \Phi^{\prime \prime}(\Psi(z))\left(\Psi^{\prime}(z)\right)^{2}+z \Phi^{\prime}(\Psi(z)) \Psi^{\prime \prime}(z)$. Evaluating at $z=1$, we obtain the mean of $m_{1}$ as $\Psi^{\prime}(1)=1 /(1-\theta)$. For $\Psi^{\prime \prime}(1)$, we have

$$
\Psi^{\prime \prime}(1)=\frac{\theta(2-\theta)}{(1-\theta)^{3}} .
$$

Hence, the variance of $m_{1}$ is

$$
\Psi^{\prime \prime}(1)+\Psi^{\prime}(1)-\left(\Psi^{\prime}(1)\right)^{2}=\theta /(1-\theta)^{3} .
$$

The sum $L$ conditional on $m_{0}$ of a Poisson branching process is known to follow the Borel-Tanner distribution (18) (see Kingman [22]; Nirei [25, 26]). Applying the Stirling's formula for the factorial $\left(\ell-m_{0}\right)$ ! in (18), we obtain (19).

\section{B Distribution of $L$}

Toward the full characterization of $d \log P_{t}$, we need to pin down the distribution of $m_{0}$ and the unconditional distribution of $L$. To do so, we need to investigate the direct effect of the firm that draws a Calvo shock. Its effect differs from the effect of a firm which reprices at the extensive margin. First, its repricing size is not $\log q$. This difference can be safely ignored when $n$ is large. However, the different repricing size leads to the second difference: its impact on the extensive margin which defines the distribution of $m_{0}$. This point can be formally analyzed as follows.

Suppose firm $i$ is hit by the Calvo event and reprices from $P_{i, t}$ to $P_{i, t}^{*}$. The size of price jump is $\Delta \log P_{i, t}=\log p^{*}-\log p_{i, t}$. Thus, we obtain that the decline of $s_{j, t}$ for 
$j \neq i$ caused by the increase in $\log P_{i, t}$ is equal to $\epsilon_{o}\left(s_{i}\right) / n$, where

$$
\epsilon_{o}\left(s_{i}\right) \equiv \frac{1}{\log q} \frac{p^{* 1-\eta}-p_{i, t}^{1-\eta}}{1-\eta} .
$$

This implies that firm $i$ 's price adjustment has an impact on the state of other firms with an order of magnitude $1 / n$. This shift in $s_{j, t}$ causes firm $j$ to adjust its price if $s_{j, t}$ is located close enough to the adjustment threshold. Namely, firms in $\left(0, \epsilon_{o}\left(s_{i}\right) / n\right]$ will adjust their prices as an optimal response to the price change by $i$. The probability for firm $j$ to adjust is $F\left(\epsilon_{o}\left(s_{i}\right) / n\right)$, where $F(\cdot)$ denotes the cumulative distribution function of $s$. Since there are $n-1$ firms that are affected by the initial price change by $i$, the number of firms that adjust due to the price change by $i$ follows a binomial distribution with population $n-1$ and probability $F\left(\epsilon_{o}\left(s_{i}\right) / n\right)$. This is the distribution of $m_{0}$.

When $n$ tends to infinity, the binomial distribution of $m_{0}$ above asymptotes to a Poisson distribution. Since $\epsilon_{o}$ has an order of magnitude $1 / n, n F\left(\epsilon_{o}\left(s_{i}\right) / n\right)$ tends to

$$
\theta_{\epsilon_{o}\left(s_{i}\right)} \equiv \frac{f_{o}}{\log q} \frac{p^{* 1-\eta}-p_{i, t}^{1-\eta}}{1-\eta}
$$

where $f_{o}$ denotes the density of firms at the repricing threshold. Then, when $n$ tends to infinity and given $\epsilon_{o}\left(s_{i}\right), m_{0}$ asymptotically follows a Poisson distribution with mean $\theta_{\epsilon_{o}\left(s_{i}\right)}$. Given the distribution of $m_{0}$, the distribution of $L$ conditional on the state $s_{i}$ of the firm that draws a Calvo shock is obtained as follows.

Lemma 3. As $n \rightarrow \infty, L$ conditional on $s_{i}$ asymptotically follows:

$$
\operatorname{Pr}\left(L=\ell \mid s_{i}\right)=\frac{\theta_{\epsilon_{o}\left(s_{i}\right)}}{\ell !} e^{-\left(\theta \ell+\theta_{\epsilon_{o}\left(s_{i}\right)}\right)}\left(\theta \ell+\theta_{\epsilon_{o}\left(s_{i}\right)}\right)^{\ell-1}
$$

Proof: A profile of state $\left(s_{i, t}\right)_{i=1}^{n}$ is drawn randomly from a joint density function $f^{n}$. $L_{t}>0$ holds only when a firm draws a Calvo event in $t$. The firm's price adjustment affects the profile $s$, and the other firms' repricing continues until $s_{i, t} \in(0,1]$ is achieved. 
This new profile constitutes a momentary equilibrium in $t . L_{t}$ is the number of firms that are involved in the repricing within the instance $t$. We compute $L_{t}$ by a best response dynamics proposed by Nirei [26] as follows.

Suppose that $i$ is hit by a Calvo event, and all the other firms' $s_{j, t}$ are reduced by $\epsilon_{o}\left(s_{i}\right) / n$. If there are no firms with $s_{j, t}$ in the range $\left(0, \epsilon_{o} / n\right]$, the adjustment process stops and the equilibrium price distribution is obtained. If there are some firms in the range, each of them adjusts the price from $p_{j, t}$ to $p_{t}^{*}$. The impact of the price change by $j$ on other firms is calculated similarly to the case of firm $i$, and denoted by $\epsilon_{1} / n$. Noting that $p_{j, t} \in\left(\underline{p}, \underline{p}+\left(\log p^{*}-\log \underline{p}\right) \epsilon_{o} / n\right), p_{j, t}$ converges to $\underline{p}$ as $n \rightarrow \infty$. Thus, when $n$ is large,

$$
\epsilon_{1}=\frac{p^{* 1-\eta}-p_{j, t}^{1-\eta}}{(1-\eta) \log q} \underset{n \rightarrow \infty}{\rightarrow} \frac{\varphi(q, 1-\eta) \underline{p}^{1-\eta}}{\log q} .
$$

Let $m_{1}$ denote the number of firms with $s_{j, t} \in\left(\epsilon_{o} / n,\left(\epsilon_{o}+\epsilon_{1}\right) / n\right] . m_{1}$ follows a binomial distribution with population $n-m_{1}$ and probability $F\left(\left(\epsilon_{o}+\epsilon_{1}\right) / n\right)-F\left(\epsilon_{o} / n\right)$.

The shift of $s_{k, t}$ by the price adjustments of $m_{1}$ firms may cause further adjustments. The adjustment process is formulated as a best response dynamics $m_{u}$ for $u=0,1, \ldots, U$ as defined in Section 3.1. The sum $L=\sum_{u=0}^{U} m_{u}$ conditional on $m_{0}$ has been already obtained as in (18). We combine (18) with that $m_{0}$ follows a Poisson distribution with mean $\theta_{\epsilon_{o}}$.

$$
\begin{aligned}
\operatorname{Pr}(L=\ell) & =\sum_{m_{0}=0}^{\ell} \frac{\theta_{\epsilon_{o}}^{m_{0}} e^{-\theta_{\epsilon_{o}}}}{m_{0} !} \frac{m_{0} !}{\ell} \frac{e^{-\theta \ell}(\theta \ell)^{\ell-m_{0}}}{\left(\ell-m_{0}\right) !} \\
& =\frac{\theta_{\epsilon_{o}} e^{-\theta \ell-\theta_{\epsilon_{o}}}}{\ell} \sum_{m_{0}=1}^{\ell} \frac{(\theta \ell)^{\ell-m_{0}} \theta_{\epsilon_{o}}^{m_{0}-1}}{\left(\ell-m_{0}\right) !\left(m_{0}-1\right) !} \\
& =\frac{\theta_{\epsilon_{o}}}{\ell !} e^{-\left(\theta \ell+\theta_{\epsilon_{o}}\right)}\left(\theta \ell+\theta_{\epsilon_{o}}\right)^{\ell-1} .
\end{aligned}
$$

This completes the proof. 


\section{Proof of Proposition 5: For sufficiently large $n$ and in a range of sufficiently} large $\pi$, the variance of inflation $d \log P_{t}$ is increasing in $\pi$.

Using Lemma 3, we derive the variance of $d \log P$. The main determinants of the variance, $L$ and $\log q$, have been already established. What remains to show is that the quantitative role played by the Calvo shock directly is negligible.

In each instance, there may be a firm that reprices due to the Calvo shock. Conditional on that a firm does reprice due to the Calvo shock, $m_{0}$ denotes the number of firms that reprice due to the decreased relative price caused by the firm that reprices due to the Calvo shock. Asymptotically as $n \rightarrow \infty, m_{0}$ follows a Poisson distribution with mean $\theta_{0}$. The probability generating function of the total number of firms that adjust at the instance of the exogenous repricing event is $\Psi_{0}(z) \equiv E\left[\Psi^{m_{0}}(z)\right]$. Note that $m_{0}$ follows a Poisson distribution with mean $\theta_{\epsilon_{o}(s)}$, in which we make it explicit that $\epsilon_{o}$ depends on $s$ which follows a distribution function $f(s)$. Then, the probability generating function is solved as $\Psi_{0}(z)=\int_{0}^{1} e^{\theta_{\epsilon_{o}(s)}(\Psi(z)-1)} f(s) d s$. The derivative with respect to $z$ yields:

$$
\begin{aligned}
& \Psi_{0}^{\prime}(z)=\int_{0}^{1} \Psi^{\prime}(z) e^{\theta_{\epsilon_{o}(s)}(\Psi(z)-1)} \theta_{\epsilon_{o}(s)} f(s) d s \\
& \Psi_{0}^{\prime \prime}(z)=\int_{0}^{1}\left(\Psi^{\prime \prime}(z) e^{\theta_{\epsilon_{o}(s)}(\Psi(z)-1)} \theta_{\epsilon_{o}(s)}+e^{\theta_{\epsilon_{o}(s)}(\Psi(z)-1)}\left(\Psi^{\prime}(z) \theta_{\epsilon_{o}(s)}\right)^{2}\right) f(s) d s .
\end{aligned}
$$

Thus, we obtain that $\Psi_{0}^{\prime}(1)=E\left[\theta_{\epsilon_{o}(s)}\right] /(1-\theta)$ and $\Psi_{0}^{\prime \prime}(1)=E\left[\theta_{\epsilon_{o}(s)}\right] \theta(2-\theta) /(1-\theta)^{3}+$ $E\left[\theta_{\epsilon_{o}(s)}^{2}\right] /(1-\theta)^{2}$.

The aggregate price $\log P_{t}$ follows a compound Poisson process with intensity rate $\mu$ and a jump size which is the multiple of individual repricing size $\log q$ and the total number of the firms that adjust in the branching process $L_{t}$.

The number of firms that draw such events in a unit time follows a Poisson distribution with mean $\mu n$. Let $X$ denote the total number of firms repricing in a 
unit time and $Y$ a random variable following the Poisson distribution with mean $\mu n$. Note that $L$ follows the same distribution as $X$ conditional on $Y=1$. Then, $V(X)=E[V(X \mid Y)]+V(E[X \mid Y])=E[Y] V(L)+V(Y) E[L]^{2}=\mu n E\left[L^{2}\right]$. Thus, the variance of $d \log P_{t}$ in a unit time is $(\log q)^{2} \mu E\left[L^{2}\right] / n$. Now, we have

$$
E\left[L^{2}\right]=E[L(L-1)]+E[L]=\Psi_{0}^{\prime \prime}(1)+\Psi_{0}^{\prime}(1)=\frac{\theta_{0}}{(1-\theta)^{3}}+\frac{E\left[\theta_{\epsilon_{o}(s)}^{2}\right]}{(1-\theta)^{2}}
$$

where $\theta_{0} \equiv E\left[\theta_{\epsilon_{o}(s)}\right]$.

First, we will show that $\theta_{0} /(1-\theta)$ is increasing in $\pi$. Using $E\left[p_{i, t}^{1-\eta}\right]=1$, we have

$$
\theta_{0}=\frac{E\left[p^{* 1-\eta}-p_{i, t}^{1-\eta}\right]}{(1-\eta) \log q} \frac{\log q}{\varphi(q, \mu / \pi)}=\frac{\varphi\left(p^{*}, 1-\eta\right)}{\varphi(q, \mu / \pi)} .
$$

Then,

$$
\begin{aligned}
\frac{\varphi\left(p^{*}, 1-\eta\right)}{\varphi(q, \mu / \pi)} & =\frac{1}{\varphi(q, \mu / \pi)}\left(\frac{\underline{\underline{p}}^{1-\eta}\left(q^{1-\eta}-1\right)}{1-\eta}+\frac{\underline{p}^{1-\eta}-1}{1-\eta}\right) \\
& =\frac{1}{\varphi(q, \mu / \pi)}\left(\frac{\varphi(q, \mu / \pi)}{\varphi(q, 1-\eta+\mu / \pi)} \varphi(q, 1-\eta)+\frac{\frac{\varphi(q, \mu / \pi)}{\varphi(q, 1-\eta+\mu / \pi)}-1}{1-\eta}\right) \\
& =\theta-\frac{1}{\eta-1}\left(\frac{1}{\varphi(q, 1-\eta+\mu / \pi)}-\frac{1}{\varphi(q, \mu / \pi)}\right) \\
& =\theta-\frac{1}{\eta-1} \int_{1-\eta+\mu / \pi}^{\mu / \pi} \frac{\partial}{\partial x} \frac{-1}{\varphi}(q, x) d x \\
& =\theta-\frac{q^{x_{8}} \log \left(q^{x_{8}}\right)-q^{x_{8}}+1}{\left(q^{x_{8}}-1\right)^{2}} \quad \text { where } x_{8} \in[1-\eta+\mu / \pi, \mu / \pi] .
\end{aligned}
$$


Now we use

$$
\begin{aligned}
\log \theta & =\log \varphi(q, 1-\eta)-\log \varphi(q, 1-\eta+\mu / \pi) \\
& =\int_{1-\eta+\mu / \pi}^{1-\eta} \frac{\varphi_{x}}{\varphi}(q, x) d x \\
& =\int_{1-\eta+\mu / \pi}^{1-\eta} \frac{\log q}{1-q^{-x}}-\frac{1}{x} d x \\
& \sim\left(\frac{\log q}{1-q^{\eta-1}}-\frac{1}{1-\eta}\right) \frac{-\mu}{\pi} \\
& \sim \frac{-\mu}{\eta-1} \frac{1}{\pi} .
\end{aligned}
$$

Then,

$$
\begin{aligned}
\frac{\theta_{o}}{1-\theta} & \sim \frac{e^{-\mu /(\pi(\eta-1))}-1+\frac{(\eta-1) \log q}{q^{\eta-1}}}{1-e^{-\mu /(\pi(\eta-1))}} \\
& \sim \frac{1-\mu /(\pi(\eta-1))-1+\frac{(\eta-1) \log q}{q^{\eta-1}}}{-\mu /(\pi(\eta-1))} \\
& \sim 1+\frac{(\eta-1)^{2}}{\mu} \frac{\pi \log q}{q^{\eta-1}} .
\end{aligned}
$$

Now, we have

$$
\frac{d}{d \pi} \frac{\pi \log q}{q^{\eta-1}}=\frac{\log q}{q^{\eta-1}}+(q-(\eta-1) \log q) q^{-\eta} \pi \frac{d \log q}{d \pi}
$$

Since $q$ dominates $\log q$ for large $q$ and since $\log q$ is increasing in $\pi$, the above expression is positive. Thus, $\theta_{o} /(1-\theta)$ is asymptotically increasing in $\pi$. Moreover, $1 /(1-\theta)^{2}$ increases unboundedly as $\pi \rightarrow \infty$. Hence, $\theta_{o} /(1-\theta)^{3}$ is asymptotically increasing in $\pi$.

Next, we show that $E\left[\theta_{\epsilon_{o}}^{2}\right] /(1-\theta)^{2}$ either grows monotonically or converges to 0 . The proof is not insightful and deferred to the next section. Combining these results, $E\left[\theta_{\epsilon_{o}(s)}\right] /(1-\theta)^{3}+E\left[\theta_{\epsilon_{o}(s)}^{2}\right] /(1-\theta)^{2}$ increases as $\pi$ increases for sufficiently large $\pi$. 


\section{Analysis of $E\left[\theta_{\epsilon_{o}(s)}\right]$ (Not for publication)}

First we derive $E\left[q^{a s}\right]$ for an arbitrary parameter $a$.

$$
\begin{aligned}
\int_{0}^{1} q^{a s} \frac{q^{s \mu / \pi}(\mu / \pi) \log q}{q^{\mu / \pi}-1} d s & =\frac{\log q}{\varphi(q, \mu / \pi)} \frac{\left.q^{s(a+\mu / \pi)}\right|_{0} ^{1}}{(a+\mu / \pi) \log q} \\
& =\frac{\varphi(q, a+\mu / \pi)}{\varphi(\mu / \pi)}
\end{aligned}
$$

Note that

$$
\begin{aligned}
\theta_{\epsilon_{o}} & =\frac{f_{o}}{\log q} \frac{p^{* 1-\eta}-p_{i t}^{1-\eta}}{1-\eta} \\
& =\frac{(\underline{p} q)^{1-\eta}}{\varphi(q, \mu / \pi)} \frac{1-q^{(s-1)(1-\eta)}}{1-\eta} \\
& =\frac{q^{1-\eta}}{\varphi(q, 1-\eta+\mu / \pi)} \frac{1-q^{(s-1)(1-\eta)}}{1-\eta} .
\end{aligned}
$$

Then,

$$
\begin{aligned}
E\left[\theta_{\epsilon_{o}}^{2}\right]= & \left(\frac{q^{1-\eta}}{(1-\eta) \varphi(q, 1-\eta+\mu / \pi)}\right)^{2}\left(1-2 q^{\eta-1} E\left[q^{s(1-\eta)}\right]+q^{2(\eta-1)} E\left[q^{2(1-\eta) s]}\right)\right. \\
= & \left(\frac{q^{1-\eta}}{(1-\eta) \varphi(q, 1-\eta+\mu / \pi)}\right)^{2} \\
& \times\left(1-2 q^{\eta-1} \frac{\varphi(q, 1-\eta+\mu / \pi)}{\varphi(q, \mu / \pi)}+q^{2(\eta-1)} \frac{\varphi(q, 2(1-\eta)+\mu / \pi)}{\varphi(q, \mu / \pi)}\right) \\
\sim & q^{2(1-\eta)}-2 \frac{q^{1-\eta}}{(\eta-1) \varphi(\mu / \pi)}+\frac{\varphi(q, 2(1-\eta)+\mu / \pi)}{\varphi(q, \mu / \pi)} \\
\sim & \frac{1}{2(\eta-1) \varphi(q, \mu / \pi)}
\end{aligned}
$$

Thus,

$$
\begin{aligned}
\frac{E\left[\theta_{\epsilon_{o}}^{2}\right]}{(1-\theta)^{2}} & \sim \frac{1}{2(\eta-1) \varphi(q, \mu / \pi)}\left(\frac{1}{-\mu /((1-\eta) \pi)}\right)^{2} \\
& =\frac{(\eta-1) \pi / \mu}{2\left(q^{\mu / \pi}-1\right)}
\end{aligned}
$$


Now we investigate the asymptotic behavior of $(\log q) / \pi$. Define $g(\pi) \equiv e^{(\log q) / \pi}$. The right-hand side of (28) is

$$
c_{2} \frac{\varphi(q, \mu / \pi) \varphi(q,-\mu / \pi)}{\pi^{2}}=c_{2} \frac{\left(g^{\mu}-1\right)\left(1-g^{-\mu}\right)}{\mu^{2}}
$$

where $c_{2}>0$ is a constant. Using this, we obtain

$$
\begin{aligned}
\frac{d}{d \pi}\left(\frac{\log q}{\pi}\right) & =\frac{d \log q}{d \pi} \frac{1}{\pi}-\frac{\log q}{\pi^{2}} \\
& =\frac{1}{\pi}\left(\frac{d \log q}{d \pi}-\frac{\log q}{\pi}\right) \\
& \sim \frac{1}{\pi}\left(c_{2} \frac{\left(g^{\mu}-1\right)\left(1-g^{-\mu}\right)}{\mu^{2}}-\log g\right) .
\end{aligned}
$$

Thus, $d g / d \pi=g(d / d \pi)((\log q) / \pi)$ satisfies:

$$
\frac{d g}{d \pi} \frac{\pi}{g} \sim c_{2} \frac{\left(g^{\mu}-1\right)\left(1-g^{-\mu}\right)}{\mu^{2}}-\log g .
$$

Namely, as $\pi$ increases, $g$ can either grow more than a constant rate, stay constant, or decreases monotonically. Therefore, $E\left[\theta_{\epsilon_{0}}^{2}\right] /(1-\theta)^{2}$ either grows monotonically or converges to 0 .

\section{References}

[1] Daron Acemoglu, Vasco M. Carvalho, Asuman Ozdaglar, and Alireza TahbazSalehi. The network origins of aggregate fluctuations. Econometrica, 80:19772016, 2012.

[2] Christian Ahlin and Mototsugu Shintani. Menu costs and Markov inflation: A theoretical revision with new evidence. Journal of Monetary Economics, 54:753784, 2007. 
[3] Fernando Alvarez, Martin Beraja, Martin Gonzalez-Rozada, and Andy Neumeyer. From hyperinflation to stable prices: Argentina's evidence on menu cost models, 2016.

[4] Fernando Alvarez and Francesco Lippi. Price setting with menu cost for multiproduct firms. Econometrica, 82:89-135, 2014.

[5] Guido Ascari and Argia M. Sbordone. The macroeconomics of trend inflation. Journal of Economic Literature, 52:679-739, 2014.

[6] Per Bak, Kan Chen, José Scheinkman, and Michael Woodford. Aggregate fluctuations from independent sectoral shocks: Self-organized criticality in a model of production and inventory dynamics. Ricerche Economiche, 47:3-30, 1993.

[7] Laurence Ball. Why does high inflation raise inflation uncertainty? Journal of Monetary Economics, 29:371-388, 1992.

[8] Laurence Ball, N. Gregory Mankiw, and David Romer. The New Keynesian economics and the output-inflation trade-off. Brookings Papers on Economic Activity, $1: 1-65,1988$.

[9] Laurence M. Ball. The case for four percent inflation. Central Bank Review, 13:17-31, 2013.

[10] Ariel Burstein and Christian Hellwig. Welfare costs of inflation in a menu cost model. American Economic Review, 98:438-443, 2008.

[11] Ricardo J. Caballero and Eduardo M. R. A. Engel. Dynamic (S,s) economies. Econometrica, 59:1659-1686, 1991. 
[12] Andrew Caplin and John Leahy. Aggregation and optimization with statedependent pricing. Econometrica, 65:601-625, 1997.

[13] Andrew S. Caplin and Daniel F. Spulber. Menu cost and the neutrality of money. Quarterly Journal of Economics, 102:703-726, 1987.

[14] Leif Danziger. A dynamic economy with costly price adjustments. American Economic Review, 89:878-901, 1999.

[15] N. Nergiz Dincer and Barry Eichengreen. Central bank transparency and independence: Undates and new measures. International Journal of Central Banking, 10:189-253, 2014.

[16] Michael Dotsey, Robert G. King, and Alexander L. Wolman. State-dependent pricing and the general equilibrium dynamics of money and output. Quarterly Journal of Economics, 114:655-690, 1999.

[17] Stanley Fischer and Franco Modigliani. Towards an understanding of the real effects and costs of inflation. Review of World Economics, 114:810-833, 1978.

[18] Xavier Gabaix. The granular origins of aggregate fluctuations. Econometrica, 79:733-772, 2011.

[19] Etienne Gagnon. Price setting during low and high inflation: Evidence from Mexico. Quarterly Journal of Economics, 124:1221-1263, 2009.

[20] Mikhail Golosov and Robert E. Lucas, Jr. Menu costs and Phillips curves. Journal of Political Economy, 115:171-196, 2007.

[21] Ruth Judson and Athanasios Orphanides. Inflation, volatility and growth. International Finance, 2:117-138, 1999. 
[22] J. F. C. Kingman. Poisson Processes. Oxford, NY, 1993.

[23] Virgiliu Midrigan. Menu costs, multiproduct firms, and aggregate fluctuations. Econometrica, 79:1139-1180, 2011.

[24] Emi Nakamura and Jón Steinsson. Monetary non-neutrality in a multisector menu cost model. Quarterly Journal of Economics, 125:961-1013, 2010.

[25] Makoto Nirei. Threshold behavior and aggregate fluctuation. Journal of Economic Theory, 127:309-322, 2006.

[26] Makoto Nirei. An interaction-based foundation of aggregate investment fluctuations. Theoretical Economics, 10:953-985, 2015.

[27] Arthur M. Okun. The mirage of steady inflation. Brookings Papers on Economic Activity, 1971:485-498, 1971.

[28] José A. Scheinkman and Michael Woodford. Self-organized criticality and economic fluctuations. American Economic Association, Papers and Proceedings, 84:417-421, 1994.

[29] Eytan Sheshinski and Yoram Weiss. Inflation and costs of price adjustment. Review of Economic Studies, 44:287-303, 1977.

[30] Nancy L. Stokey. The Economics of Inaction. Princeton, 2009. 